KEK-TH-1029

ROM2F/2005/15

hep-th/0508003

\title{
D-branes in a Big Bang/Big Crunch Universe: Misner Space
}

\author{
Yasuaki Hikida, ${ }^{a *}$ Rashmi R. Nayak ${ }^{b \dagger}$ and Kamal L. Panigrahi ${ }^{b \ddagger}$ \\ ${ }^{a}$ Theory Group, High Energy Accelerator Research Organization (KEK) \\ Tukuba, Ibaraki 305-0801, Japan \\ b Dipartimento di Fisica \& INFN, Sezione di Roma 2, "Tor Vergata", \\ Roma 00133, Italy
}

\begin{abstract}
We study D-branes in a two-dimensional Lorentzian orbifold $\mathbb{R}^{1,1} / \Gamma$ with a discrete boost $\Gamma$. This space is known as Misner or Milne space, and includes big crunch/big bang singularity. In this space, there are D0-branes in spiral orbits and D1-branes with or without flux on them. In particular, we observe imaginary parts of partition functions, and interpret them as the rates of open string pair creation for D0-branes and emission of winding closed strings for D1-branes. These phenomena occur due to the time-dependence of the background. Open string $2 \rightarrow 2$ scattering amplitude on a D1-brane is also computed and found to be less singular than closed string case.
\end{abstract}

\footnotetext{
*E-mail: hikida@post.kek.jp

${ }^{\dagger}$ E-mail: Rashmi.Nayak@roma2.infn.it

${ }^{\ddagger}$ E-mail: Kamal.Panigrahi@roma2.infn.it
} 


\section{Contents}

1 Introduction $\quad 2$

2 Closed strings in Misner space $\quad 3$

2.1 Closed strings in the twisted sectors . . . . . . . . . . . . . . . . . . 4

2.2 Torus amplitude . . . . . . . . . . . . . . . . . . . . 5

2.3 No-ghost theorem . . . . . . . . . . . . . . . . 8

3 D-branes and annulus amplitudes $\quad 10$

3.1 Classical analvsis on D-branes . . . . . . . . . . . . . . . . . . . 10

3.2 Localized D-brand. . . . . . . . . . . . . . . . . . . . 13

3.2.1 Annulus amplitudd . . . . . . . . . . . . . . . . 13

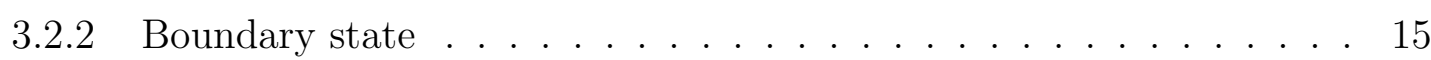

3.2.3 Open string pair creation . . . . . . . . . . . . . . . . . . . . 16

3.3 Wrapping D-brand . . . . . . . . . . . . . . . . . . . . 19

3.3.1 Annulus amplitude . . . . . . . . . . . . . . . . . . . . . 19

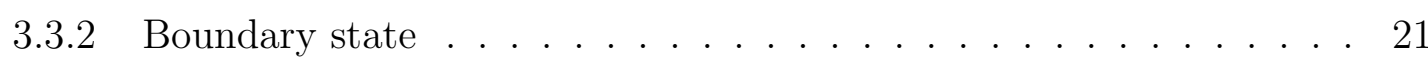

3.3.3 Emission of winding closed strings . . . . . . . . . . . . . . . . . . 22

4 Superstrings and D-branes in Misner space $\quad 24$

4.1 Torus amplitude. . . . . . . . . . . . . . . . . . . 24

4.2 Localized D-brand . . . . . . . . . . . . . . . . . . . . . . 26

4.3 Wrapping D-brand . . . . . . . . . . . . . . . . . . . . . . . . 29

5 Correlation functions $\quad 31$

5.1 Wave function and two point function . . . . . . . . . . . . . . . 31

5.2 Three point function . . . . . . . . . . . . . . . . 32

5.3 Four point function . . . . . . . . . . . . . . . . . . 33

$6 \quad$ Strings and D-branes in Grant space $\quad 36$

6.1 Torus amplitude. . . . . . . . . . . . . . . . . 36

6.2 Annulus amplitudes . . . . . . . . . . . . . . . . . . 38

6.3 Four point function . . . . . . . . . . . . . . . . . . . . . 40

$\begin{array}{lll}7 & \text { Conclusion } & 41\end{array}$

\begin{tabular}{|ll} 
A Theta and eta functions & 43
\end{tabular} 


\section{Introduction}

The study of string theory on cosmological backgrounds is of great interest in view of understanding the stringy phenomena, such as, in the early universe cosmology. Around the initial space-like singularity at the beginning of the universe, the field theory techniques cease to be valid, and the string effects therefore should be taken into account. However, despite of much progress in string theory on time-independent backgrounds, this important aspect has not been widely challenged. This is mainly due to the shortage of tractable models for strings in cosmological or time-dependent backgrounds. A few of known ways are to utilize the orbifold method and the gauged WZW models; A limited list of references on this subject is [1, 2, 3, 4, 5, 6, 9, 7, 8, 10, 11, for the Lorentzian orbifolds and [12, 13, 14, 15, 16, for the coset models.

In this paper, we consider a simplest Lorentzian orbifold $\mathbb{R}^{1,1} / \Gamma$, which is two dimensional flat Minkowski space-time identified by a discrete boost $\Gamma$. This model describes strings in Misner (Milne) space [17, which has been studied in [4, 18, 19, 20, 21, 22. Misner space includes big crunch/big bang singularity [17, 23, and hence the behavior of strings at the singularity is of interest. However, this space also has regions where closed time-like curves (CTCs) exist, which might invalidate the theory on this background. Because of this subtlety, the authors of [5, 6] studied the parabolic orbifold $\mathbb{R}^{1,2} / \Gamma$, which is the three dimensional Minkowski space-time with the identification of a discrete null boost. The orbifold admits supersymmetry, light-like Killing vectors and closed null curves, but no CTC. Even though these good properties, the orbifold is known to be unstable due to the large back reaction [8, 9, 10, 11]. Therefore, it might be enough to study the simpler model to examine the properties of the big crunch/big bang singularities in string models. In fact, the Misner space case seems more interesting, since we can expect particle (string) productions, which do not occur in the parabolic orbifold with light-like Killing vectors.

In a Euclidean orbifold, strings in twisted sectors play an important role to resolve the orbifold singularity, thus it is natural to expect that the big crunch/big bang singularity is also resolved by condensation of closed strings in twisted sectors. These twisted strings are investigated in [19, 20, 21, 22] by emphasizing the similarity to open strings in the presence of electric flux, though any clear conclusion on the resolution (if any) cannot be made. This is mainly because the closed strings in twisted sectors are not localized in the fixed point as seen below. Interestingly, it was conjectured in 24 that the twisted closed string condensation removes the regions with CTC as the stringy version of chronology protection [25]. Recent related works are given in [26, 27, 28, 29, 30, 31, 32, 33.

The aim of this paper is to investigate D-branes in Misner space. ${ }^{1}$ Since there are

\footnotetext{
${ }^{1}$ This is a work subsequent to [34, where we investigated classical aspects of D-branes in another big bang/big crunch universe, i.e., D-branes in the Nappi-Witten gauged WZW model. D-branes in other time-dependent backgrounds are investigated, for example, in [35, 36, 37, 38, 39.
} 
D0-branes and D1-branes in the covering space (two dimensional Minkowski space), there are also D0-branes and D1-branes in the two dimensional orbifold theory. In general, D-branes behave very interestingly in curved backgrounds contrary to D-branes in flat space. In particular, we observe closed string emission from a D-brane falling into the stack of NS5-branes [40] using the boundary state formalism [41, 42, 43, 44, 45]. Since our background is time-dependent and includes the big bang/big crunch singularity, we expect the similar emissions of strings happen on the D-branes. For the purpose, we compute the annulus amplitudes for open strings, and examine the emissions of strings from the imaginary parts of the amplitudes. Another interesting observation may be made in the open string scattering amplitude. It was shown in [18] that the $2 \rightarrow 2$ scattering amplitude diverges due to the graviton exchange, which is the signal of the large back-reaction. Since there is no graviton sector in the open string spectrum, it is worth examining open string scatterings and study (would-be) divergences.

The layout of the paper is as follows. In section 2 we review briefly some aspects of closed strings in Misner space for bosonic strings. In particular, we examine the spectrum in the twisted sectors and compute the torus amplitude. We also show the no-ghost theorem for the closed strings in the twisted sectors. In section 3, we first present the effective field theory analysis on D-branes and write down the classical configurations of both the D0 and D1-branes. We next compute annulus amplitudes for open strings on a D0-brane and a D1-brane. We also construct the boundary states for the D-branes and reproduce the amplitudes as scattering amplitudes between boundary states. There are imaginary parts of the annulus amplitudes, which indicate the instability of the D-branes. We see that open strings between image branes are pair-created on the D0-brane and winding closed strings are emitted from the D1-brane. In section 4, we generalize this results to superstring settings, and check that the emissions are not related to tachyonic states. In section 5, we turn our attention to the scattering process, and analyze the divergence structures of four point functions. We find that the open string four point function is less singular than closed string one. In section 6, we investigate open strings and D-branes in so-called Grant space [46], which should behave less singular than Misner space case due to the introduction of an extra non-trivial dimension. Finally in section

7 we present the summary of our result and a set of open questions deserved to be investigated. We list up the formulae for theta and eta functions in appendix A.

\section{Closed strings in Misner space}

Misner space can be regarded as a quotient of a two dimensional flat Minkowski spacetime by a Lorentz boost

$$
x^{ \pm} \sim e^{ \pm 2 \pi \gamma} x^{ \pm}
$$


with $\gamma \in \mathbb{R}$. The space includes four regions divided by the lines $x^{+} x^{-}=0$. Among the four, the two regions $x^{+} x^{-}>0$ are called as the cosmological regions. Using the coordinate transformation $x^{ \pm}=\frac{1}{\sqrt{2}} t e^{ \pm \gamma \psi}$, the metric is given by

$$
d s^{2}=-d t^{2}+\gamma^{2} t^{2} d \psi^{2}
$$

with the periodicity $\psi \sim \psi+2 \pi$. Therefore, the universe consists of a circle with timedependent radius. The universe starts with an infinite large radius and shrinks as time goes. At $t=0$, the radius becomes zero, where the point may be interpreted as the big crunch singularity. Another region starts at $t=0$ with big bang singularity, expands for a while and ends with an infinite large radius. There are two other regions $x^{+} x^{-}<0$, which are called as whisker regions. The metric in these regions is given by

$$
d s^{2}=d r^{2}-\gamma^{2} r^{2} d \chi^{2}
$$

with $x^{ \pm}= \pm \frac{1}{\sqrt{2}} r e^{ \pm \gamma \chi}$. Due to the identification (2.1), the time coordinate has the periodicity $\chi \sim \chi+2 \pi$, and hence the universe includes closed time-like curves (CTCs) everywhere in the whisker regions. One may find in [23] arguments on more detailed structure of this universe and singularity.

\subsection{Closed strings in the twisted sectors}

Because of the identification (2.1), the spectrum includes sectors where

$$
X^{ \pm}(\tau, \sigma+2 \pi)=e^{ \pm 2 \pi \gamma w} X^{ \pm}(\tau, \sigma)
$$

with $w \in \mathbb{Z}$. Since the spectrum in the untwisted sector $(w=0)$ is almost the same as the usual flat case, we will concentrate on the twisted sectors $(w \neq 0)$. In the Misner space, there are two types of non-trivial cycles; $\psi$ direction in the cosmological regions and $\chi$ direction in the whisker regions. Therefore the closed strings may wrap either of the cycles.

In order to catch the physical picture of winding strings, we restrict to the lowest modes as

$$
X^{ \pm}(\tau, \sigma)= \pm \sqrt{\frac{\alpha^{\prime}}{2}} \frac{\alpha_{0}^{ \pm}}{\nu} e^{ \pm \nu(\tau+\sigma)} \mp \sqrt{\frac{\alpha^{\prime}}{2}} \frac{\tilde{\alpha}_{0}^{ \pm}}{\nu} e^{\mp \nu(\tau-\sigma)}
$$

with $\nu=\gamma w$. The Virasoro constraint restricts the values

$$
\omega^{2}=\frac{1}{2}\left(\alpha_{0}^{+} \alpha_{0}^{-}+\alpha_{0}^{-} \alpha_{0}^{+}\right), \quad \tilde{\omega}^{2}=\frac{1}{2}\left(\tilde{\alpha}_{0}^{+} \tilde{\alpha}_{0}^{-}+\tilde{\alpha}_{0}^{-} \tilde{\alpha}_{0}^{+}\right)
$$

as

$$
\omega^{2}=\frac{\alpha^{\prime}}{4} \vec{k}^{2}+N-\frac{d}{24}+\frac{1}{2} \nu^{2}, \quad \quad \tilde{\omega}^{2}=\frac{\alpha^{\prime}}{4} \vec{k}^{2}+\tilde{N}-\frac{d}{24}+\frac{1}{2} \nu^{2}
$$


Here we have included $d$ extra flat dimension, and denoted $\vec{k}$ as the momenta and $N, \tilde{N}$ as the occupation number operators. In case $\omega=\tilde{\omega}$, we may set

$$
\alpha_{0}^{+}=\alpha_{0}^{-}=\epsilon \omega, \quad \tilde{\alpha}_{0}^{+}=\tilde{\alpha}_{0}^{-}=\tilde{\epsilon} \omega
$$

where we take $\epsilon, \tilde{\epsilon}= \pm 1$, though only two choices are essentially different. If we take $\epsilon=\tilde{\epsilon}=1$, then we obtain

$$
X^{ \pm}(\tau, \sigma)=\frac{\sqrt{2 \alpha^{\prime}} \omega}{\nu} e^{ \pm \nu \sigma} \sinh \nu \tau
$$

This represents a string wrapping $\psi$ direction and localized in the cosmological regions. Another choice may be $\epsilon=-\tilde{\epsilon}=1$, which leads to

$$
X^{ \pm}(\tau, \sigma)= \pm \frac{\sqrt{2 \alpha^{\prime}} \omega}{\nu} e^{ \pm \nu \sigma} \cosh \nu \tau
$$

This string winds $\chi$ direction and is localized in a whisker region. See [19, 20, 21, 22] for physical properties of these winding strings.

\subsection{Torus amplitude}

We re-derive the torus amplitude computed in [3, 4] in order to clarify the way how to compute annulus amplitudes. First we use the path integral formalism. We specify the worldsheet coordinate with Euclidean signature as $(z, \bar{z})$ where $z=\sigma_{1}+i \sigma_{2}$ with periodic boundary conditions $\sigma_{1} \sim \sigma_{1}+2 \pi$ and $\sigma_{2} \sim \sigma_{2}+2 \pi$. The worldsheet metric is given by

$$
d s^{2}=\left(d \sigma_{1}+\tau d \sigma_{2}\right)\left(d \sigma_{1}+\bar{\tau} d \sigma_{2}\right)
$$

Due to the identification (2.1), we can assign the boundary conditions

$$
X^{ \pm}\left(\sigma_{1}+2 \pi, \sigma_{2}\right)=e^{ \pm 2 \pi \gamma w} X^{ \pm}\left(\sigma_{1}, \sigma_{2}\right), \quad X^{ \pm}\left(\sigma_{1}, \sigma_{2}+2 \pi\right)=e^{ \pm 2 \pi \gamma k} X^{ \pm}\left(\sigma_{1}, \sigma_{2}\right)
$$

with $w, k \in \mathbb{Z}$. Since the untwisted sector $(w, k)=(0,0)$ is almost the same as in the flat

space case, we focus on the twisted sector $(w, k) \neq(0,0)$. The general solution satisfying the above boundary conditions is

$$
X^{ \pm}\left(\sigma_{1}, \sigma_{2}\right)=e^{ \pm \gamma\left(w \sigma_{1}+k \sigma_{2}\right)}\left(\sum_{m, n \in \mathbb{Z}} a_{m, n}^{ \pm} e^{i\left(m \sigma_{1}+n \sigma_{2}\right)}\right)
$$

Thus the eigenvalue of the Laplacian operator is

$$
\Delta=\frac{1}{\tau_{2}^{2}}\left(\partial_{2}-\tau \partial_{1}\right)\left(\partial_{2}-\bar{\tau} \partial_{1}\right)=-\frac{1}{\tau_{2}^{2}}(n-\tau m+y)(n-\bar{\tau} m-\bar{y})
$$

with $y\left(\equiv y_{1}+i y_{2}\right)=i \gamma(\tau w-k)$. 
Performing integral with respect to $a_{m, n}$, we obtain

$$
\mathcal{T}_{w, k}^{-1}(\tau)=\operatorname{Det}(-\Delta)=\prod_{m, n} \frac{1}{\tau_{2}^{2}}(n-\tau m+y)(n-\bar{\tau} m-\bar{y}) .
$$

In order to obtain a finite expression, we use the zeta function regularization. ${ }^{2}$ First we find

$$
\prod_{m} \frac{1}{\tau_{2}^{2}}=\lim _{s \rightarrow 0}\left(\frac{1}{\tau_{2}^{2}}\right)^{2\left(\sum_{m \geq 1} m^{-s}\right)+1}=\left(\frac{1}{\tau_{2}^{2}}\right)^{2 \zeta(0)+1}=1 .
$$

In the last equality, we have used $\zeta(0)=-1 / 2$. Next we observe

$$
\prod_{m, n}(n-\tau m+y)(n-\bar{\tau} m-\bar{y})=\prod_{n}(n+y)(n-\bar{y}) \prod_{m \neq 0, n}(n-\tau m+y)(n-\bar{\tau}-\bar{y}) .
$$

Using the formula

$$
\prod_{n=-\infty}^{\infty}(n+a)=a \prod_{n=1}^{\infty}\left(-n^{2}\right)\left(1-\frac{a^{2}}{n^{2}}\right)=2 i \sin \pi a
$$

we can compute as

$$
\prod_{n}(n+y)(n-\bar{y})=4 \sin \pi y \sin \pi \bar{y}
$$

and

$$
\begin{aligned}
& \prod_{m \neq 0, n}(n-\tau m+y)(n-\bar{\tau} m-\bar{y}) \\
& =\prod_{m \geq 1} e^{-\pi i(\tau m-y)} e^{\pi i(\bar{\tau} m+\bar{y})}\left(1-q z^{-1}\right)\left(1-\bar{q} \bar{z}^{-1}\right) e^{-\pi i(\tau m+y)} e^{\pi i(\bar{\tau} m-\bar{y})}(1-q z)(1-\bar{q} \bar{z}) .
\end{aligned}
$$

Here we have denoted as $q=\exp (2 \pi i \tau)$ with $\tau=\tau_{1}+i \tau_{2}$ and $z=\exp (2 \pi i y)$. Applying the generalized zeta function regularization

$$
\sum_{n=1}^{\infty}(n+\theta)=\lim _{s \rightarrow-1} \sum_{n=1}^{\infty}(n+\theta)^{-s}=-\frac{1}{12}+\frac{1}{2} \theta(1-\theta),
$$

we obtain

$$
\prod_{m \geq 1} e^{-\pi i(\tau m-y)} e^{\pi i(\bar{\tau} m+\bar{y})}=e^{2 \pi \tau_{2} \sum_{m \geq 1}\left(m+\frac{i y_{1}}{\tau_{2}}\right)}=e^{-\frac{\pi \tau_{2}}{6}+i \pi y_{1}+\frac{\pi y_{1}^{2}}{\tau_{2}}} .
$$

Combining everything, we reproduce the partition function given in [4] as

$$
\mathcal{T}_{w, k}(\tau)=\operatorname{Det}^{-1}(-\Delta)=\left|\frac{\eta(\tau)}{\vartheta_{1}(y \mid \tau)}\right|^{2} e^{-\frac{2 \pi y_{1}^{2}}{\tau_{2}}}
$$

\footnotetext{
${ }^{2}$ See, e.g., 47, 48.
} 
Summing over all twisted sectors $(w, k) \neq(0,0),{ }^{3}$ we obtain the modular invariant partition function under $\tau \rightarrow \tau+1$ and $\tau \rightarrow-1 / \tau$. Notice that the winding numbers are interchanged $(w, k) \rightarrow(k,-w)$ under the S-transformation $\tau \rightarrow-1 / \tau$.

This result can be obtained also in the oscillator formalism. In the $w$-th twisted sector (2.4), the mode expansion is given by

$$
X^{ \pm}(\tau, \sigma)=i \sqrt{\frac{\alpha^{\prime}}{2}} \sum_{n}\left[\frac{\alpha_{n}^{ \pm}}{n \pm i \nu} e^{-i(n \pm i \nu)(\tau+\sigma)}+\frac{\tilde{\alpha}_{n}^{ \pm}}{n \mp i \nu} e^{-i(n \mp i \nu)(\tau-\sigma)}\right]
$$

with $n \in \mathbb{Z}$ and $\nu=\gamma w$. The oscillators satisfy the following commutation relations

$$
\left[\alpha_{m}^{+}, \alpha_{n}^{-}\right]=(-m-i \nu) \delta_{m+n}, \quad\left[\tilde{\alpha}_{m}^{+}, \tilde{\alpha}_{n}^{-}\right]=(-m+i \nu) \delta_{m+n} .
$$

In terms of oscillators, the Virasoro generators are written as

$$
\begin{aligned}
& L_{0}=\frac{1}{2} i \nu(1-i \nu)-\sum_{n \geq 1} \alpha_{-n}^{+} \alpha_{n}^{-}-\sum_{n \geq 0} \alpha_{-n}^{-} \alpha_{n}^{+}, \\
& \tilde{L}_{0}=\frac{1}{2} i \nu(1-i \nu)-\sum_{n \geq 0} \tilde{\alpha}_{-n}^{+} \tilde{\alpha}_{n}^{-}-\sum_{n \geq 1} \tilde{\alpha}_{-n}^{-} \tilde{\alpha}_{n}^{+}
\end{aligned}
$$

for the Misner space part. As in [3, 4, we have set $\alpha_{0}^{-}$and $\tilde{\alpha}_{0}^{+}$as creation operators and $\alpha_{0}^{+}$and $\tilde{\alpha}_{0}^{-}$as annihilation operators. ${ }^{45}$

When computing the partition function, it is convenient to define the occupation number operators $N, \tilde{N}$ and the boost operators $J, \tilde{J}$ as

$$
\begin{aligned}
& N=\sum_{n \geq 1}\left(n N_{n}^{+}+n N_{n}^{-}\right), \quad \tilde{N}=\sum_{n \geq 1}\left(n \tilde{N}_{n}^{+}+n \tilde{N}_{n}^{-}\right), \\
& i J=\sum_{n \geq 1} N_{n}^{+}-\sum_{n \geq 0} N_{n}^{-}, \quad i \tilde{J}=\sum_{n \geq 0} \tilde{N}_{n}^{+}-\sum_{n \geq 1} \tilde{N}_{n}^{-},
\end{aligned}
$$

where the oscillator number operators are

$$
N_{n}^{ \pm}=-\frac{1}{n \mp i \nu} \alpha_{-n}^{ \pm} \alpha_{n}^{\mp}, \quad \quad \tilde{N}_{n}^{ \pm}=-\frac{1}{n \pm i \nu} \tilde{\alpha}_{-n}^{ \pm} \tilde{\alpha}_{n}^{\mp}
$$

Then we can reproduce the partition function (2.23) by

$$
\begin{aligned}
\mathcal{T}(\tau) & =\sum_{\{k, w\} \neq\{0,0\}} \mathcal{T}_{k, w}(\tau) \\
\mathcal{T}_{k, w}(\tau) & =\operatorname{Tr}_{w} g^{k} q^{L_{0}-\frac{1}{12}} \bar{q}^{\tilde{L}_{0}-\frac{1}{12}}=(q \bar{q})^{-\frac{1}{12}+\frac{1}{2} i \nu(1-i \nu)} \operatorname{Tr}_{w} e^{2 \pi \gamma k i(J+\tilde{J})} q^{\nu J+N} \bar{q}^{-\nu \tilde{J}+\tilde{N}} .
\end{aligned}
$$

${ }^{3}$ The full torus amplitude includes the untwisted sector, and the contribution from the sector is modular invariant by itself.

${ }^{4}$ For $\nu<0$, we set $\alpha_{0}^{+}$and $\tilde{\alpha}_{0}^{-}$as creation operators and $\alpha_{0}^{-}$and $\tilde{\alpha}_{0}^{+}$as annihilation operators oppositely to $\nu>0$ case. The following equations are modified accordingly.

${ }^{5}$ This definition of Hilbert space is actually different from the previous one, and it is interpreted as a Wick rotation. In fact, we can reproduce the torus amplitude computed in the path integral as shown below. The relation will be discussed in more detail in the next subsection. See also [19]. 
The trace $\operatorname{Tr}_{w}$ is taken over the Hilbert space of $w$-th twisted sector. The projection operator $\sum_{k} g^{k}$ with $g=\exp (2 \pi i \gamma(J+\tilde{J}))$ is needed to project the Hilbert space into subspace invariant under the discrete boost (2.1). Notice that the twist number $k$ corresponds to the winding number $k$ along $\sigma_{2}$ cycle in the path integral formalism (see (2.13) $)$.

In order to obtain the expression in a critical string theory, we add contributions from extra $d$ flat directions ( $d=24$ for bosonic string theory) and ghost part. Then, we obtain the partition function as ${ }^{6}$

$$
\mathcal{T}=\sum_{k, w} \int_{\mathcal{F}} \frac{d^{2} \tau}{4 \pi^{2} \alpha^{\prime} \tau_{2}^{2}} \frac{e^{-2 \pi \tau_{2} \gamma^{2} w^{2}}}{\left(4 \pi^{2} \alpha^{\prime} \tau_{2}\right)^{\frac{d-2}{2}}\left|\vartheta_{1}(i \gamma(w \tau+k) \mid \tau) \eta(\tau)^{d-3}\right|^{2}},
$$

where $\mathcal{F}$ denotes the fundamental region $\left(\left|\tau_{1}\right|<\frac{1}{2},|\tau|>1\right)$.

\subsection{No-ghost theorem}

In this subsection, we show no-ghost theorem for the spectrum of closed strings in the $w$-th twisted sector. For simplicity, we consider critical bosonic string theory with $d=24$, and focus on the left-moving part. ${ }^{7}$ We define the Hilbert space by the action of the creation modes $\alpha_{-n}^{ \pm}, \alpha_{-n}^{i}$ with $n \geq 1$ and ghost modes $c_{-n}$ with $n \geq 0$ and $b_{-n}$ with $n \geq 1$. We use the vacuum state satisfying

$$
\frac{1}{2}\left(\alpha_{0}^{+} \alpha_{0}^{-}+\alpha_{0}^{-} \alpha_{0}^{+}\right)\left|\omega^{2}, \vec{k}\right\rangle=\omega^{2}\left|\omega^{2}, \vec{k}\right\rangle, \quad \vec{\alpha}_{0}\left|\omega^{2}, \vec{k}\right\rangle=\sqrt{\frac{\alpha^{\prime}}{2}} \vec{k}\left|\omega^{2}, \vec{k}\right\rangle, \quad b_{0}\left|\omega^{2}, \vec{k}\right\rangle=0
$$

with $\omega^{2} \in \mathbb{C}$. An important property of the vacuum state is

$$
\alpha_{0}^{ \pm}\left|\omega^{2}, \vec{k}\right\rangle \propto\left|\omega^{2} \pm i \nu, \vec{k}\right\rangle
$$

which means that the action of the zero modes is just the shift of the eigenvalue $\omega^{2}$. The physical states are then constructed as the cohomology of the BRST operator

$$
Q_{B}=\sum_{n} c_{n} L_{-n}+\sum_{m, n} \frac{(m-n)}{2}: c_{m} c_{n} b_{-m-n}:-c_{0} .
$$

In other words, the physical states satisfy $Q_{B} \psi=0$ with equivalent relation $\psi \sim \psi+Q_{B} \chi$. In order to show the no-ghost theorem, we will see that the physical Hilbert space is isomorphic to $\mathcal{H}^{\perp}$ which has no ghost and light-cone excitations.

It is convenient to introduce filtration degree counted by ${ }^{8}$

$$
N_{f}=-\sum_{n \neq 0} \frac{1}{n+i \nu} \alpha_{-n}^{-} \alpha_{n}^{+}+\sum_{n=1}^{\infty}\left(c_{-n} b_{n}-b_{-n} c_{n}\right)
$$

\footnotetext{
${ }^{6}$ We will neglect volume factors, such as, $i V_{d}$ in the case of $d$ dimensional Minkowski space-time.

${ }^{7}$ The left-moving part of the spectrum of twisted closed string is almost the same as that of open strings between D-branes with different electric flux.

${ }^{8}$ We mainly follow the analysis in [49, 50]. For discussions on no-ghost theorem for related models, see, e.g., [51, 2, 52].
} 
then we can split the BRST operator by the degree

$$
Q_{B}=Q_{0}+Q_{1}+Q_{2}, \quad\left[N_{f}, Q_{j}\right]=j Q_{j}
$$

As shown in 53, 54, non-trivial cohomology of $Q_{0}$ is isomorphic to non-trivial cohomology of $Q_{B}$. Therefore, it is enough for our purpose to show that the cohomology of $Q_{0}(=$ $\left.\sum_{n \neq 0} c_{n} \alpha_{-n}^{+} \alpha_{0}^{-}\right)$is isomorphic to $\mathcal{H}^{\perp}$. As in [49, 50, we define

$$
R=\sum_{n \neq 0} b_{n} \alpha_{-n}^{-}
$$

which gives

$$
S=\left\{Q_{0}, R\right\}=-\sum_{n \geq 1}\left\{(n-i \nu)\left[N_{n}^{-}+b_{-n} c_{n}\right]+(n+i \nu)\left[N_{n}^{+}+c_{-n} b_{n}\right]\right\} \alpha_{0}^{-} .
$$

If $S|\psi\rangle=s \alpha_{0}^{-}|\psi\rangle$ with $s \neq 0$, then we have

$$
\left|\psi^{\prime}\right\rangle\left(\equiv \alpha_{0}^{-}|\psi\rangle\right)=\frac{1}{s}\left\{Q_{0}, R\right\}|\psi\rangle=\frac{1}{s} Q_{0} R|\psi\rangle
$$

for $Q_{0}|\psi\rangle=0$ and hence $Q_{0}\left|\psi^{\prime}\right\rangle=0$. Therefore, non-trivial cohomology exists only if $s=0$, which means that the non-trivial cohomology is isomorphic to $\mathcal{H}^{\perp}$. A crucial difference from the usual flat space case is that now the zero modes $\alpha_{0}^{ \pm}$do not commute with each other. However, thanks to the definition of vacuum (2.31) and its property (2.32), we can mimic the analysis in flat space case [49, 50] as seen above.

Next, let us see this observation is consistent with the one-loop amplitude (2.30). In order to do so, we will use the identity

$$
\frac{1}{2 \sin T}=i \sum_{n=0}^{\infty} e^{-i(2 n+1) T}=\int_{-\infty}^{\infty} d\left(\omega^{2}\right) \rho\left(\omega^{2}\right) e^{-2 \omega^{2} T}
$$

where

$$
\rho\left(\omega^{2}\right)=\frac{1}{2}\left(1+\tanh \pi \omega^{2}\right) .
$$

This equality is valid only when $0<T<1$, but we assume this is true also for the other T. ${ }^{9}$ The most important property of $\rho\left(\omega^{2}\right)$ is that it is invariant under $\omega^{2} \rightarrow \omega^{2}+i n$ with $n \in \mathbb{Z}$. Using the identity (2.39) with $T=\pi(i \gamma(w \tau+k))$ and $\rho\left(\omega^{2}+i n\right)=\rho\left(\omega^{2}\right)$, the one-loop amplitude can be rewritten as

$$
\begin{aligned}
\mathcal{T} & =\sum_{k, w} \int_{\mathcal{F}} \frac{d^{2} \tau}{\tau_{2}} \int \frac{d^{24} k}{(2 \pi)^{24}} e^{-\pi \tau_{2} \alpha^{\prime} \vec{k}^{2}-2 \pi \tau_{2} \gamma^{2} w^{2}}\left|\int d\left(\omega^{2}\right) \rho\left(\omega^{2}\right) \sum_{\text {states }} e^{2 \pi i \gamma k\left(J-\omega^{2}\right)} q^{\nu\left(J-\omega^{2}\right)+N-1}\right|^{2} \\
& =\sum_{k, w} \int_{\mathcal{F}} \frac{d^{2} \tau}{\tau_{2}} \int \frac{d^{24} k}{(2 \pi)^{24}} e^{-\pi \tau_{2} \alpha^{\prime} \vec{k}^{2}-2 \pi \tau_{2} \gamma^{2} w^{2}}\left|\frac{1}{2 \sin \pi(i \gamma(w \tau+k))} \sum_{\text {states } \in \mathcal{H}^{\perp}} q^{N-1}\right|^{2} \cdot(2.41)
\end{aligned}
$$

\footnotetext{
${ }^{9}$ Notice that we use a different $\rho\left(\omega^{2}\right)$ from [19] such that there is a valid region for real $T$. The difference may originate from different regularization schemes.
} 
The last expression implies that negative norm states do not propagate in the one-loop amplitude.

Here we should comment on the interpretation of (2.39). As seen in the torus amplitude, the left hand side of (2.39) may be interpreted as the contribution of zero modes, which are treated as creation and annihilation modes. In a sense, this definition of vacuum is for a Wick rotated model, since the definition is the same as the one in Euclidean orbifolds, such as, in the $\mathbb{C} / \mathbb{Z}_{N}$ orbifold. On the other hand, the right hand side may be interpreted as the contribution of zero modes, where the vacuum state is defined by (2.31). Thus, it is natural to regard $\rho\left(\omega^{2}\right)$ as the density of state in the Lorentzian model itself (not in the Wick rotated one).

\section{D-branes and annulus amplitudes}

The main purpose of this paper is to investigate how D-branes behave in timedependent backgrounds, particularly in the presence of big crunch/big bang singularity. Since we have D0-branes and D1-branes in the covering space, we can construct D0-branes and D1-branes even in the orbifold model as seen in the next subsection. In subsection 3.2. we compute one-loop amplitude for open strings on the D0-brane, and identify that the imaginary part of the amplitude as the pair creation rate of open strings. Then, we examine D1-brane in subsection 3.3. where annulus amplitude is computed and the imaginary part is identified as the emission rate of closed strings.

\subsection{Classical analysis on D-branes}

In the two dimensional Minkowski space-time $\mathbb{R}^{1,1}$, we have D0-brane passing from infinite past to infinite future, and D1-brane with or without constant gauge flux on its world-volume. Since the Misner space is regarded as a orbifold of $\mathbb{R}^{1,1}$, D-branes in Misner space may be obtained by utilizing the orbifold method. We will also find the D0-brane trajectory and the consistent gauge flux on D1-brane by directly solving the DBI actions on the D-branes as in [34].

D0-brane in the covering space is given by a straight line

$$
x^{ \pm}=x_{0}^{ \pm}+p^{ \pm} u
$$

with a parameter $u \in \mathbb{R}$. Using the coordinate transformations given above and removing the parameter $u$, this line can be expressed as

$$
t \sinh (\gamma \psi+A)=C, \quad e^{A}=\sqrt{\frac{p^{-}}{p^{+}}}, \quad C=\sqrt{\frac{p^{+} p^{-}}{2}}\left(\frac{x_{0}^{+}}{p^{+}}-\frac{x_{0}^{-}}{p^{-}}\right)
$$

for the cosmological regions, and

$$
r \cosh (\gamma \tau+A)=C
$$




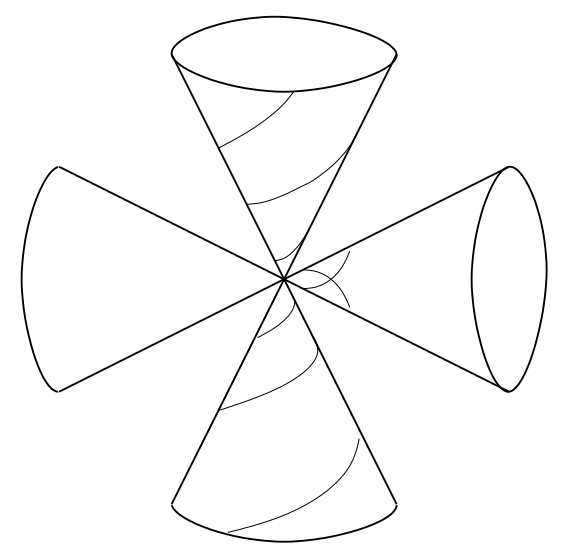

(A) D0-brane

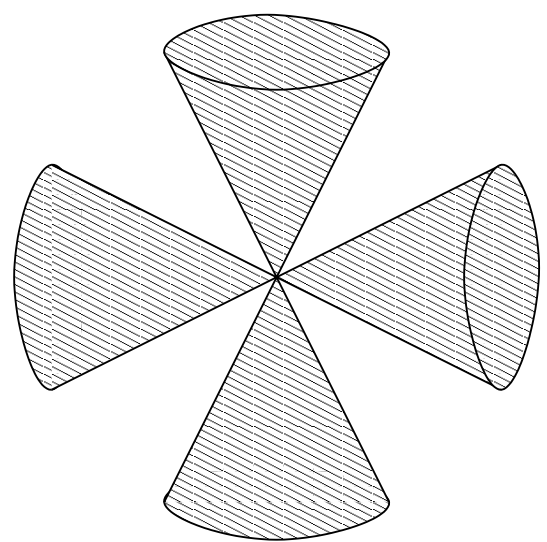

(B) D1-brane

Figure 1: (A) D0-brane travels from infinite past to infinite future by passing a whisker region. (B) D1-brane covers the whole Misner space with or without flux on it.

with the same $A, C$ for the whisker regions. In the orbifold model, we have to sum up all trajectories with $A \rightarrow A+2 \pi \gamma k$ with $k \in \mathbb{Z}$. Thus, the D0-brane starts from far past in the cosmological region $t \sim-\infty$ and approaches spirally into the big crunch singularity. Then it crosses a whisker region and goes to the other cosmological region (see fig. (1).

The DBI action for effective theory on the D0-brane in the cosmological regions is

$$
S=-\tau_{0} \int d t \sqrt{1-\gamma^{2} t^{2} \dot{\psi}^{2}}, \quad \dot{\psi}=\frac{d \psi}{d t} .
$$

The normalization includes the inverse of the string coupling $\tau_{0} \propto g_{s}^{-1}$. From the equation of motion, we obtain

$$
P_{\psi}=\frac{\delta \mathcal{L}}{\delta \dot{\psi}}=\frac{\tau_{0} \gamma^{2} t^{2} \dot{\psi}}{\sqrt{1-\gamma^{2} t^{2} \dot{\psi}}}, \quad \quad \dot{\psi}^{2}=\frac{1}{\gamma^{2} t^{2}\left(1+\frac{\tau_{0}^{2}}{P_{\psi}^{2}} \gamma^{2} t^{2}\right)}
$$

which is consistent with (3.2) if $C^{2}=\frac{P_{\psi}^{2}}{\gamma^{2} \tau_{0}^{2}}$. The DBI action in the whisker regions is

$$
S=-\tau_{0} \int d \chi \sqrt{\gamma^{2} r^{2}-\dot{r}^{2}}, \quad \quad \dot{r}=\frac{d r}{d \chi} .
$$

Since the energy is conserved, we obtain

$$
E=\frac{\delta \mathcal{L}}{\delta \dot{r}} \dot{r}-\mathcal{L}=\frac{\tau_{0} \gamma^{2} r^{2}}{\sqrt{\gamma^{2} r^{2}-\dot{r}^{2}}}, \quad \quad \dot{r}^{2}=\gamma^{2} r^{2}-\frac{\tau_{0}^{2}}{E^{2}} \gamma^{4} r^{4},
$$

which is consistent with (3.3) if $C^{2}=\frac{E^{2}}{\gamma^{2} \tau_{0}^{2}}$.

For D1-brane, we can construct D-brane wrapping the whole Misner space, and add a constant gauge flux on it in the covering space. In the cosmological regions with the coordinate system (2.2), we have the DBI action

$$
S=-\tau_{1} \int d t d \psi \sqrt{\gamma^{2} t^{2}-F_{t \psi}^{2}} .
$$


Thus the gauge flux can be computed from the Gauss constraint

$$
\frac{\delta \mathcal{L}}{\delta F_{t \psi}}=\frac{\tau_{1} F_{t \psi}}{\sqrt{\gamma^{2} t^{2}-F_{t \psi}^{2}}}=\Pi, \quad F_{t \psi}^{2}=\frac{\gamma^{2} t^{2}}{1+\frac{\tau_{1}^{2}}{\Pi^{2}}} .
$$

In the whisker regions, similar analysis leads to the gauge field

$$
F_{\chi r}^{2}=\frac{\gamma^{2} r^{2}}{1+\frac{\tau_{1}^{2}}{\Pi^{2}}}
$$

Using the relations

$$
F_{t \psi}=-\gamma t F_{+-}, \quad F_{\chi r}=-\gamma r F_{+-},
$$

we can see that the time-dependent gauge flux (3.9) and (3.10) in this coordinate system corresponds to constant flux in $x^{ \pm}$coordinate system, which is natural in the covering space. Because of this fact, we can solve the open string theory on D1-brane even with the time-dependent gauge flux using the orbifold method.

Due to the non-trivial gauge flux on D-branes, open strings feel the background metric in a modified way [55]. We denote the induced closed string metric on D-brane as $g_{a b}$ and the closed string coupling as $g_{s}$. Then, open string metric $G_{a b}$ and open string coupling $G_{s}$ in a configuration with $\mathcal{F}=B+F$ are given by

$$
G_{a b}=g_{a b}-\mathcal{F}_{a c} g^{c d} \mathcal{F}_{d b}, \quad G_{s} \equiv e^{\Phi_{o}}=g_{s} \sqrt{\frac{-\operatorname{det} G}{-\operatorname{det}(g+\mathcal{F})}} .
$$

The open string metrics are computed as

$$
d s^{2}=\frac{1}{1+\frac{\Pi^{2}}{\tau_{1}^{2}}}\left(-d t^{2}+\gamma^{2} t^{2} d \psi^{2}\right), \quad d s^{2}=\frac{1}{1+\frac{\Pi^{2}}{\tau_{1}^{2}}}\left(d r^{2}-\gamma^{2} r^{2} d \chi^{2}\right)
$$

and the open string coupling is $G_{s}=g_{s} / \sqrt{1+\Pi^{2} / \tau_{1}^{2}}$, which differ from closed string ones by total normalization. The low energy spectra for open strings may be read from the eigenfunctions of Laplacians in terms of open string quantities:

$$
\Delta=\frac{1}{e^{-\Phi_{o}} \sqrt{-G}} \partial_{a} e^{-\Phi_{o}} \sqrt{-G} G^{a b} \partial_{b}
$$

Since the open strings see the same metric and string coupling up to normalization, the open string spectrum is the same as the closed string one. This is consistent with the fact that the open string spectrum is not changed by introduction of constant gauge flux in the covering space.

The parameter

$$
\Theta^{i j}=-\left(\frac{1}{g+\mathcal{F}} \mathcal{F} \frac{1}{g-\mathcal{F}}\right)^{i j}
$$


which represents the non-commutativity of space-time, can be computed as

$$
\left(\Theta^{t \psi}\right)^{2}=\frac{\tau_{1}^{4}}{\gamma^{2} t^{2} \Pi^{4}}\left(1+\frac{\Pi^{2}}{\tau_{1}^{2}}\right), \quad\left(\Theta^{\chi r}\right)^{2}=\frac{\tau_{1}^{4}}{\gamma^{2} r^{2} \Pi^{4}}\left(1+\frac{\Pi^{2}}{\tau_{1}^{2}}\right)
$$

Thus we can see that the effective theory has time-dependent non-commutativity. Notice that this theta parameter is again constant in the covering space due to the relations

$$
\Theta^{t \psi}=-\frac{1}{\gamma t} \Theta^{+-}, \quad \Theta^{\chi r}=-\frac{1}{\gamma r} \Theta^{+-},
$$

and hence we can solve the effective theory by utilizing the results with a constant noncommutative parameter. This is quite interesting fact since there are only few solvable models with time-dependent non-commutativity.

\subsection{Localized D-brane}

We first deal with $\mathrm{D} p$-branes localized in the spatial direction of the Misner space part. Utilizing the orbifold construction, we can construct the D-brane by summing up all image branes in the covering space. More explicitly, we put a D0-brane in two dimensional Minkowski space-time at $e^{2 \pi a} x^{+}+b=e^{-2 \pi a} x^{-}-b$ with $a, b \in \mathbb{R}$, then the $k^{-}$

th image branes are at $e^{2 \pi(a+\gamma k)} x^{+}+b=e^{-2 \pi(a+\gamma k)} x^{-}-b$. Speaking differently, we have to consider all open strings stretched between these mirror branes. First we compute annulus amplitude for open strings between these branes, and then construct boundary state for the D-brane. We find the imaginary part of the one-loop amplitude, which implies the open string pair creation between these image branes.

\subsubsection{Annulus amplitude}

Let us compute annulus amplitude for open strings on a D-brane localized in the spatial direction of Misner space. Since there may be a subtlety in the definition of spectrum as mentioned above, we first derive it in the path integral formalism. Later we will check that the oscillator formalism gives the same result. As in the closed string case, we use Euclidean worldsheet $\left(\sigma_{1}, \sigma_{2}\right)$ with $0 \leq \sigma_{1} \leq \pi$ and $\sigma_{2} \sim \sigma_{2}+2 \pi$, whose metric is

$$
d s^{2}=d \sigma_{1}^{2}+t^{2} d \sigma_{2}^{2}
$$

The boundary condition in this case is

$$
\partial_{\sigma_{1}}\left(e^{2 \pi(a+\gamma k)} X^{+}+e^{-2 \pi(a+\gamma k)} X^{-}\right)=0, \quad \partial_{\sigma_{2}}\left(e^{2 \pi(a+\gamma k)} X^{+}-e^{-2 \pi(a+\gamma k)} X^{-}\right)=0
$$

at $\sigma_{1}=0$ and those replaced with $k \leftrightarrow k^{\prime}$ at $\sigma_{1}=\pi$. Moreover, the boundaries of the worldsheet must be on the branes

$$
e^{2 \pi(a+\gamma k)} X^{+}+b=e^{-2 \pi(a+\gamma k)} X^{-}-b
$$


at $\sigma=0$ and those with $k \leftrightarrow k^{\prime}$ at $\sigma=\pi$. The mode expansions are then $\left(\nu=2 \gamma\left(k-k^{\prime}\right)\right)^{10}$

$$
\begin{aligned}
X^{ \pm}\left(\sigma_{1}, \sigma_{2}\right) & =\mp b+\sum_{m \in \mathbb{Z}} e^{\mp 2 \pi(a+\gamma k) \pm \nu \sigma_{1}+i m \sigma_{2}} a_{m, 0} \\
& +\sum_{m \in \mathbb{Z}, n>0} e^{\mp 2 \pi(a+\gamma k)+i m \sigma_{2}}\left(a_{m, n}^{ \pm} e^{i(n \mp i \nu) \sigma_{1}}+a_{m, n}^{\mp} e^{-i(n \pm i \nu) \sigma_{1}}\right) .
\end{aligned}
$$

Performing the path integral as in the closed string case, we obtain

$$
\mathcal{A}_{k, k^{\prime}}(t)=\left|\prod_{m}(m-t \nu) \prod_{m, n>0}(m-i t(n-i \nu))(m+i t(n+i \nu))\right|^{-1}=\frac{\eta(i t)}{\vartheta_{1}(t \mid \nu \| i t)} e^{-\pi t \nu^{2}} .
$$

Notice that the final expression does not depend on the parameters $a, b$ of the D0-brane orbit. $^{11}$

Next, we derive the same result in the oscillator formalism. Because of the boundary condition (3.19), the mode expansions are given by

$$
X^{ \pm}(\tau, \sigma)=\mp b+i \sqrt{\frac{\alpha^{\prime}}{2}} \sum_{n} e^{\mp 2 \pi(a+\gamma k)}\left(\frac{\alpha_{n}^{ \pm}}{n \pm i \nu} e^{-i(n \pm i \nu)(\tau+\sigma)}+\frac{\alpha_{n}^{\mp}}{n \mp i \nu} e^{-i(n \mp i \nu)(\tau-\sigma)}\right),
$$

where the commutation relations for the oscillators are $\left[\alpha_{m}^{+}, \alpha_{n}^{-}\right]=(-m-i \nu) \delta_{m+n}$. The Virasoro generator is given by ${ }^{12}$

$$
L_{0}=\frac{1}{2} i \nu(1-i \nu)+\sum_{n \geq 1} \alpha_{-n}^{+} \alpha_{n}^{-}+\sum_{n \geq 0} \alpha_{-n}^{-} \alpha_{n}^{+} .
$$

Then we find the partition function as

$$
\mathcal{A}_{k, k^{\prime}}=\operatorname{Tr}_{\mathcal{H}_{k, k^{\prime}}} e^{-2 \pi t\left(L_{0}-\frac{1}{12}\right)}=\frac{\eta(i t)}{\vartheta_{1}(t \mid \nu \| i t)} e^{-\pi t \nu^{2}},
$$

which reproduces (3.22).

Open strings on $\mathrm{D} p$-brane have other parts; ghost part and extra $d$ dimension part with $p$ directions with Neumann boundary condition and $d-p$ directions with Dirichlet boundary condition. Taking these parts into account, the full partition function is given by

$$
\mathcal{A}=\sum_{k, k^{\prime}} \int \frac{d t}{t} \frac{e^{-\pi t \nu^{2}}}{\left(8 \pi^{2} \alpha^{\prime} t\right)^{\frac{p}{2}} \vartheta_{1}(t \mid \nu \| i t) \eta(i t)^{d-3}} .
$$

\footnotetext{
${ }^{10}$ We only consider $\nu \neq 0$ case since the case with $\nu=0$ is almost the same as the flat space case.

${ }^{11}$ It may sound strange that the annulus amplitude does not depend on the D0-brane orbit since the geodesic crossing the point $x^{+}=x^{-}=0$ is incomplete [23. Thus, we may be able to say that the D0-brane does not feel the real singular point $x^{+}=x^{-}=0$ as in the case of Nappi-Witten model 34, though the precise meaning is obscure.

${ }^{12}$ As in the closed string case, we should assign for $\nu<0$ the creation and annihilation modes to the zero modes in the opposite way to $\nu>0$ case.
} 
This expression is essentially the same as obtained in [56]. Performing a modular transformation $(t \rightarrow s=1 / t)$, we can rewrite the amplitude in the closed string channel

$$
\mathcal{A}=\sum_{k, k^{\prime}} \int d s \frac{i}{\left(8 \pi^{2} \alpha^{\prime}\right)^{\frac{p}{2}} s^{\frac{d+1-p}{2}} \vartheta_{1}(i \mid \nu \| i s) \eta(i s)^{d-3}} .
$$

As seen below, we can reproduce this result as an overlap between boundary states.

\subsubsection{Boundary state}

Let us reinterpret the annulus amplitude in terms of closed strings, in other words, we rewrite the amplitude as an overlap between boundary states. In the closed string channel, we can see how closed strings couple to D-branes. Since the D0-brane is a point-like object in Misner space, winding strings do not couple to the D-brane due to its macroscopic size. We first construct boundary state for the original D0-brane in the covering space, and sum over every boundary states for image branes mapped by the discrete boost. We prepare the boundary state for the original D0-brane satisfying

$$
\left.\left(e^{ \pm 2 \pi a} \alpha_{n}^{ \pm}+e^{\mp 2 \pi a} \tilde{\alpha}_{-n}^{\mp}\right)|\tilde{D} 0\rangle\right\rangle=0
$$

for $n \neq 0$ and

$$
\sinh (2 \pi a) t+\cosh (2 \pi a) x+\sqrt{2} b=0, \quad x^{ \pm}=\frac{1}{\sqrt{2}}(t \pm x)
$$

for zero modes. The boundary state can be written explicitly in terms of oscillators as

$$
|\widetilde{D 0}\rangle\rangle=\int d t d p e^{\sum_{n \geq 1} \frac{1}{n}\left(e^{4 \pi a} \alpha_{-n}^{+} \tilde{\alpha}_{-n}^{+}+e^{-4 \pi a} \alpha_{-n}^{-} \tilde{\alpha}_{-n}^{-}\right)} e^{i p(\sinh (2 \pi a) t+\cosh (2 \pi a) x+\sqrt{2} b)}|t, p\rangle .
$$

Since the orbifold action is generated by ${ }^{13}$

$$
g=\exp (2 \pi i \gamma \hat{J}), \quad i \hat{J}=i x^{-} p^{+}-i x^{+} p^{-}+\sum_{n \geq 1}\left(N_{n}^{+}-N_{n}^{-}+\tilde{N}_{n}^{+}-\tilde{N}_{n}^{-}\right)
$$

the boundary state is expressed as

$$
\left.\left.|D 0\rangle\rangle=\sum_{k} g^{k}|\widetilde{D 0}\rangle\right\rangle=\sum_{k}|D 0, k\rangle\right\rangle
$$

where we define $(\kappa=a+k \gamma)$

$$
|D 0, k\rangle\rangle=\int d t d p e^{\sum_{n \geq 1} \frac{1}{n}\left(e^{4 \pi \kappa} \alpha_{-n}^{+} \tilde{\alpha}_{-n}^{+}+e^{-4 \pi \kappa} \alpha_{-n}^{-} \tilde{\alpha}_{-n}^{-}\right)} e^{i p(\sinh (2 \pi \kappa) t+\cosh (2 \pi \kappa) x+\sqrt{2} b)}|t, p\rangle .
$$

This may be also obtained by solving the condition for the boundary state directly.

Having the boundary state for the D0-brane, we can compute the overlap between the boundary states from

$$
\mathcal{A}_{k_{2}, k_{1}}(s)=\left\langle\left\langle D 0, k_{2}\left|e^{\pi i s\left(L_{0}+\tilde{L}_{0}-\frac{1}{12}\right)}\right| D 0, k_{1}\right\rangle\right\rangle .
$$

\footnotetext{
${ }^{13}$ The zero modes satisfy $\left[x^{ \pm}, p^{\mp}\right]=-i$.
} 
Non-trivial contribution may arise from zero mode part. In order to compute the zero mode contribution, it is convenient to rewrite as 57

$$
\frac{1}{\cosh (2 \pi \kappa)} \int d t d p e^{i p\left(\tanh (2 \pi \kappa) t+x^{\prime}\right)}|t, p\rangle, \quad x^{\prime}=x+\sqrt{2} b \operatorname{sech}(2 \pi \kappa) .
$$

Then we find

$$
\begin{aligned}
& \frac{1}{\cosh \left(2 \pi \kappa_{1}\right) \cosh \left(2 \pi \kappa_{2}\right)} \int d t d p e^{i p\left(\left[\tanh \left(2 \pi \kappa_{1}\right)-\tanh \left(2 \pi \kappa_{2}\right)\right] t+x_{1}^{\prime}-x_{2}^{\prime}\right)-\pi s \alpha^{\prime} p^{2} / 2} \\
& =\frac{1}{\cosh \left(2 \pi \kappa_{1}\right) \cosh \left(2 \pi \kappa_{2}\right)\left|\tanh \left(2 \pi \kappa_{1}\right)-\tanh \left(2 \pi \kappa_{2}\right)\right|}=\frac{1}{\sinh (\pi|\nu|)} .
\end{aligned}
$$

Combining the contribution from the non-zero modes, we obtain

$$
\mathcal{A}(s)=\frac{i e^{-\frac{\pi s}{6}}}{\sin (\pi i|\nu|) \prod_{n \geq 1}\left(1-e^{-2 \pi(n s-\nu)}\right)\left(1-e^{-2 \pi(n s+\nu)}\right)} .
$$

The full boundary state for $\mathrm{D} p$-brane is given by the product

$$
\left.\left.\left.|D p, l o c\rangle\rangle=\mathcal{N}_{p} \sum_{k}\left|B_{g h}\right\rangle\right\rangle \otimes|D 0, k\rangle\right\rangle \otimes|B p\rangle\right\rangle
$$

where $\left.\left|B_{g h}\right\rangle\right\rangle$ denotes the ghost part. For $d$ free boson part, we have

$$
|B p\rangle\rangle=e^{-\sum_{n \geq 1} \frac{1}{n}\left(\alpha_{-n}^{\beta} \tilde{\alpha}_{-n}^{\beta}-\alpha_{-n}^{I} \tilde{\alpha}_{-n}^{I}\right)} \delta^{(d-p)}(\hat{x}-x)|0\rangle,
$$

where $2 \leq \beta \leq p+1$ for the directions parallel to the $\mathrm{D} p$-brane and $p+2 \leq I \leq d+1$ for the transverse directions. The position of the $\mathrm{D} p$-brane in the transverse directions is represented as $x$. The coefficient is

$$
\mathcal{N}_{p}=2^{-\frac{d+4}{4}} \sqrt{\pi}\left(2 \pi \sqrt{\alpha^{\prime}}\right)^{\frac{d}{2}-p-1}
$$

which is also used for the boundary state of usual $\mathrm{D} p$-brane. Now the overlap between the boundary states is computed as

$$
\langle\langle D p, l o c|\Delta| D p, l o c\rangle\rangle=\frac{\alpha^{\prime}}{2 \pi} \int d s\left\langle\left\langle D p, l o c\left|e^{-\pi s\left(L_{0}+\tilde{L}_{0}-\frac{c}{12}\right)}\right| D p, l o c\right\rangle\right\rangle
$$

which can be shown to reproduce (3.27). Here we used the central charge including the ghost part $c=d-24$. If we pick up each pair of image branes, the above computation is exactly the same as in [57] as the interaction between co-moving $\mathrm{D} p$-branes.

\subsubsection{Open string pair creation}

We have obtained the one-loop amplitude in the form of integration over $t$ (3.26). However, along the integration contour, the amplitude diverges at $t=n /|\nu|$ with $n=$ $1,2, \cdots$, where $\vartheta_{1}$ in the denominator vanishes. In order to avoid the divergence, we shift 
the integration contour a little in the complex plane, which leads to an imaginary part of the partition function. Picking up the poles, the imaginary part of the amplitude may be summarized as

$$
-2 \operatorname{Im} \mathcal{A}=\sum_{k, k^{\prime}} \sum_{n=1}^{\infty} \frac{(-1)^{n+1}}{n\left(8 \pi^{2} \alpha^{\prime}\right)^{\frac{p}{2}}}\left(\frac{|\nu|}{n}\right)^{\frac{p}{2}} \sum_{\text {states }} e^{-2 \pi n \frac{N-\frac{d}{24}}{|\nu|}-\pi n|\nu|}
$$

where we have defined $\eta(i t)^{-d} \equiv \sum_{\text {states }} e^{-2 \pi t\left(N-\frac{d}{24}\right)}$.

The situation is very similar to that of [56], where the annulus amplitude of open strings between co-moving D-branes is computed. In our case, the co-moving D-branes are image branes mapped by the discrete boost in the covering space. The system of comoving D-branes is T-dual to the configuration with two D-branes with different electric flux, and the open strings between these branes are analogue to charged particles in electric flux. It is well-known that charged particles are pair created in background with electric flux by non-perturbative effect [58. It is understood that the pair creation of charged particles acts to dilute the background flux. The pair creation rate may be deduced from the imaginary part of one-loop amplitude by using the optical theorem. The open string analogue was given in [59], where the imaginary part of annulus amplitude for open strings was computed and compared with the charged particle case. In that case, open strings are pair created due to the electric flux on D-branes. In the T-dual picture, the annulus amplitude may be interpreted as the phase shift due to the scattering between co-moving D-branes. Therefore, the imaginary part of the amplitude can be interpreted as the rate that one of the D-branes is absorbed into another D-brane. The open string pair creation occurs when the D-branes are close to each other, and the effect reduces the relative velocities [56. This interpretation is T-dual to the fact that the pair creation of charged particles dilutes the background flux.

Therefore, also in our case, the imaginary part (3.42) can be interpreted as the absorption rate between image branes and also the rate of open string pair creation. Note that the density of states behaves as $\rho_{N} \sim e^{2 \pi N}$ for high energy, thus the imaginary part diverges for $n /|\nu|<1$. $^{14}$ This means that the probe D-brane localized in the spatial direction of Misner space is unstable due to the large absorption rate between mirror branes. The situation is analogous to the closed string case, where image probe particles create large black holes due to large back reaction [1].

Utilizing the analysis in 60], we can confirm the imaginary part is due to the open string pair creation, not due to the emission of closed strings (see fig. 2). With the help

\footnotetext{
${ }^{14}$ The pair of image branes with $|\nu|>1$ move faster than the speed of light relatively, which implies the instability of the brane system. This situation is T-dual to the D-branes with supercritical gauge flux, where the open strings are enlarged by the electric force and would be of infinite length.
} 

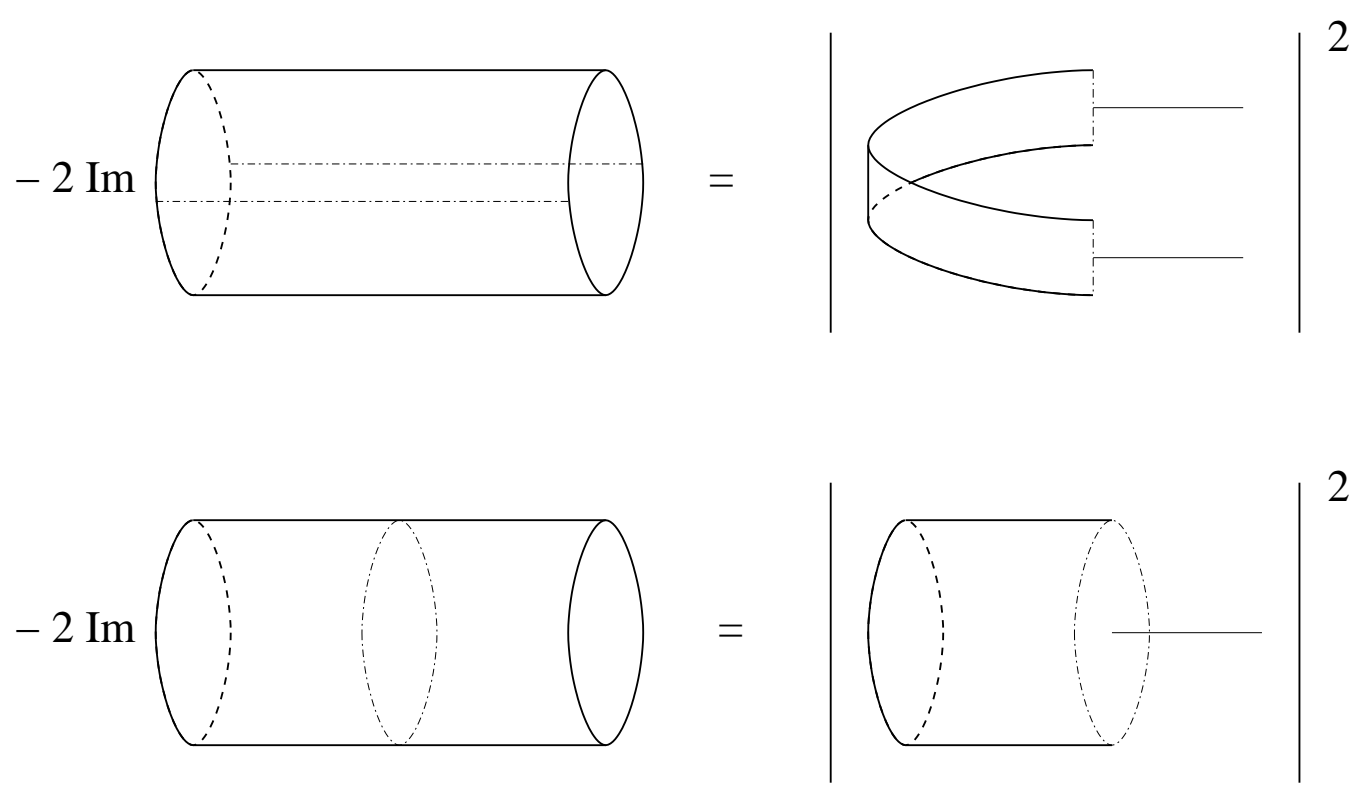

Figure 2: The imaginary part of the cylinder amplitude may be interpreted as the rate of open string pair creation or/and closed string emission from the optical theorem. We identify that the imaginary part for open strings on the D0-brane is due to open string pair creation, and that for open strings on the D1-brane is due to emission of winding closed strings.

of the identity (2.39) with $T=\pi|\nu| t$, we can rewrite the partition function (3.26) as

$$
\mathcal{A}=\sum_{k, k^{\prime}} \int_{0}^{\infty} \frac{d t}{t} \int_{-\infty}^{\infty} d\left(\omega^{2}\right) \int \frac{d^{p} k}{(2 \pi)^{p}} \sum_{\text {states }} \rho\left(\omega^{2}\right) e^{-2 \pi t\left(\omega^{2}|\nu|+\alpha^{\prime} \vec{k}^{2}+N-\frac{d}{24}+\frac{\nu^{2}}{2}\right)}
$$

Defining $\mathcal{A}=\sum_{k, k^{\prime}} \int \frac{d^{p} k}{(2 \pi)^{p}} \mathcal{A}_{k, k^{\prime}}(\vec{k})$, we obtain

$$
\begin{aligned}
-\frac{1}{2 \pi \alpha^{\prime}} \frac{d}{d \vec{k}^{2}} \mathcal{A}_{k, k^{\prime}}(\vec{k}) & =\int_{0}^{\infty} d t \int_{-\infty}^{\infty} d\left(\omega^{2}\right) \sum_{\text {states }} \rho\left(\omega^{2}\right) e^{-2 \pi t\left(\omega^{2}|\nu|+\alpha^{\prime} \vec{k}^{2}+N-\frac{d}{24}+\frac{\nu^{2}}{2}\right)} \\
& =\int_{-\infty}^{\infty} d\left(\omega^{2}\right) \sum_{\text {states }} \rho\left(\omega^{2}\right) \frac{1}{2 \pi\left(\omega^{2}|\nu|+\alpha^{\prime} \vec{k}^{2}+N-\frac{d}{24}+\frac{\nu^{2}}{2}\right)}
\end{aligned}
$$

When performing the integration of $\omega^{2}$, we encounter poles arising from the propagator $1 /\left(L_{0}-\frac{d}{24}\right)$, which corresponds to on-shell poles of open strings. These poles lead to imaginary part, therefore we can conclude that the imaginary part (3.42) is due to the open string pair creation as discussed in [56].

To check the consistence we will reproduce the previous expression (3.42). For the purpose, it is useful to notice the identity

$$
\frac{1}{2}\left(1+\tanh \pi \omega^{2}\right)=\sum_{n=1}^{\infty}(-1)^{n+1} e^{2 n \pi \omega^{2}}
$$


for $\omega^{2}<0$. Then, by picking up the poles, we obtain

$$
-\frac{1}{2 \pi \alpha^{\prime}} \frac{d}{d \vec{k}^{2}} \operatorname{Im} \mathcal{A}_{k, k^{\prime}}(\vec{k})=\frac{1}{2|\nu|} \sum_{n=1}^{\infty} \sum_{\text {states }}(-1)^{n} e^{-\frac{2 \pi n}{|\nu|}\left(\alpha^{\prime} \vec{k}^{2}+N-\frac{d}{24}+\frac{\nu^{2}}{2}\right)} .
$$

Here we have used $\rho\left(\omega^{2}+i n\right)=\rho\left(\omega^{2}\right)$ with $n \in \mathbb{Z}$. Integrating out $\vec{k}^{2}$ and $\vec{k}$, we can get the expression (3.42).

One may wonder what would happen if we use (3.27) to evaluate the imaginary part instead of (3.26). In this case, the imaginary part arises at $s=|\nu| / n$, and this is due to the Regge trajectories in the closed string spectrum. In other words, the divergence comes from the sum over the states with integer multiple of spin $2 n$. Therefore, it is not appropriate to use this channel for regarding the cause of the imaginary part as the emission of on-shell strings. ${ }^{15}$

\subsection{Wrapping D-brane}

Next we consider $\mathrm{D} p$-brane wrapping the whole Misner space including constant gauge field strength $F^{+-}=f$ in the covering space. In order to compute annulus amplitude for open strings, we have to restrict the Hilbert space into subspace invariant under the discrete boost using the projection operator $P=\sum_{k} g^{k}$ where $g X^{ \pm}=e^{ \pm 2 \pi \gamma} X^{ \pm}$. In the boundary state formalism, the winding strings can couple to the D-brane wrapping the whole Misner space. Moreover, we will find that the sum of the twisted sector corresponds to the sum over all the twist number $k$. Recasting the expression in the closed string channel, we identify that the imaginary part of the cylinder amplitude is due to the emission of winding closed strings (see fig. 2).

\subsubsection{Annulus amplitude}

We first compute the annulus amplitude in the oscillator formalism. Due to the flux $F^{+-}=f$, the boundary condition is shifted as

$$
\partial_{\sigma} X^{ \pm}= \pm f \partial_{\tau} X^{ \pm}
$$

at the boundary $\sigma=0, \pi$. Therefore, the mode expansion is given by

$$
X^{ \pm}=x^{ \pm}-2 i \alpha^{\prime} p^{ \pm} \frac{f \sigma+\tau}{\sqrt{1-f^{2}}}+i \sqrt{2 \alpha^{\prime}} \sum_{n} \frac{\alpha_{n}^{ \pm}}{n} e^{i n \tau} \cos (n \sigma \mp i \operatorname{arctanh} f)
$$

where

$$
\left[x^{ \pm}, p^{\mp}\right]=-i, \quad\left[\alpha_{m}^{+}, \alpha_{n}^{-}\right]=-m \delta_{m, n} .
$$

\footnotetext{
${ }^{15}$ Similar discussions were given in [20] for poles in one-loop amplitudes.
} 
Notice that the Virasoro generator is the same as in the case without gauge flux $(f=0)$;

$$
L_{0}=-2 \alpha^{\prime} p^{+} p^{-}-\sum_{n \geq 1} \alpha_{-n}^{+} \alpha_{n}^{-}-\sum_{n \geq 1} \alpha_{-n}^{-} \alpha_{n}^{+} .
$$

The orbifold action $g=\exp (2 \pi \gamma i \hat{J})$ is generated by using

$$
i \hat{J}=i x^{-} p^{+}-i x^{+} p^{-}+\sum_{n \geq 1}\left(N_{n}^{+}-N_{n}^{-}\right) .
$$

Now we can compute the partition function as

$$
\begin{aligned}
\mathcal{A}_{k}(t) & =\operatorname{Tr} e^{2 \pi i \nu \hat{J}} e^{-2 \pi t\left(L_{0}-\frac{1}{12}\right)} \\
& =\frac{e^{-\frac{\pi t}{6}}}{(2 i \sin (\pi i \nu))^{2} \prod_{n \geq 1}\left(1-e^{-2 \pi(n t-\nu)}\right)\left(1-e^{-2 \pi(n t+\nu)}\right)},
\end{aligned}
$$

where $\nu=\gamma k$ and we assume that $k \neq 0$. A non-trivial contribution has arisen from the zero mode sector, which can be computed as (see, e.g, [61])

$$
\int d^{2} p\left\langle\mathbf{p}\left|g^{k} q^{\alpha^{\prime} \mathbf{p}^{2}}\right| \mathbf{p}\right\rangle=\int d^{2} p \delta^{(2)}\left(\left(1-g^{k}\right) \mathbf{p}^{2}\right) q^{\alpha^{\prime} \mathbf{p}^{2}}=\operatorname{det}^{-1}\left(1-g^{k}\right)=\frac{1}{4 \sinh ^{2} \pi \nu},
$$

where we use the fact that the eigenvalues of $g^{k}$ on $p^{ \pm}$are $e^{ \pm 2 \pi \nu}$.

Next we confirm the calculation of annulus amplitude in terms of path integral, especially the zero mode contribution. As in the D0-brane case, we use Euclidean worldsheet $\left(\sigma_{1}, \sigma_{2}\right)$ with $0 \leq \sigma_{1} \leq \pi$ and $\sigma_{2} \sim \sigma_{2}+2 \pi$, where the worldsheet metric and the Laplacian are given by

$$
d s^{2}=d^{2} \sigma_{1}+t^{2} d^{2} \sigma_{2}, \quad \Delta=\frac{1}{t^{2}}\left(\partial_{2}^{2}+t^{2} \partial_{1}^{2}\right) .
$$

We assign Neumann boundary condition at the boundaries of worldsheet as

$$
\partial_{1} X^{ \pm}\left(\sigma_{1}=0, \sigma_{2}\right)=0, \quad \partial_{1} X^{ \pm}\left(\sigma_{1}=\pi, \sigma_{2}\right)=0
$$

Due to the identification (2.1), we have the twisted sector along $\sigma_{2}$ direction as

$$
X^{ \pm}\left(\sigma_{1}, \sigma_{2}+2 \pi\right)=e^{ \pm 2 \pi \gamma k} X^{ \pm}\left(\sigma_{1}, \sigma_{2}\right)
$$

The sum over $k$ corresponds to the projection operator $P=\sum_{k} g^{k}$ in the oscillator formalism. The solutions to the conditions (3.55) and (3.56) are given by

$$
X^{ \pm}\left(\sigma_{1}, \sigma_{2}\right)=\sum_{m \in \mathbb{Z}, n \geq 0} a_{m, n}^{ \pm} e^{i(m \mp i \nu) \sigma_{2}}\left(e^{i n \sigma_{1}}+e^{-i n \sigma_{1}}\right)
$$

In order to evaluate the path integral, we have to take the gauge flux into account. This is included as the boundary interaction

$$
S_{B}=\frac{1}{2 \pi \alpha^{\prime} \tau_{2}} \int_{\partial \Sigma} A_{+} d X^{+}, \quad A_{+}=F_{+-} X^{-}, \quad A_{-}=0
$$


which is cancelled between the contributions at $\sigma_{1}=0$ and $\sigma_{2}=\pi$ in the path integral. The other contributions are summarized as the inverse of

$$
\begin{aligned}
\operatorname{Det}(-\Delta) & =\left|\prod_{m}(m-i \nu)^{2} \prod_{m, n>0}(m+i t n-i \nu)(m-i t n-i \nu)\right| \\
& =(2 i \sin (\pi i \nu))^{2} \prod_{n \geq 1} e^{2 \pi n t}\left(1-e^{-2 \pi(n t-\nu)}\right)\left(1-e^{-2 \pi(n t+\nu)}\right) \\
& =2 \sinh (\pi|\nu|) \frac{\vartheta_{1}(i|\nu| \mid i t)}{i \eta(i t)}
\end{aligned}
$$

which reproduce the previous result (3.52).

To compute the full partition function, we have to include contributions from the ghosts and from $d$ free bosons. Since we assign Neumann boundary condition to $p-1$ bosons and Dirichlet boundary condition to $d_{\perp}(\equiv d+1-p)$ bosons, the annulus amplitude becomes

$$
\mathcal{A}=\sum_{k \neq 0} \int_{0}^{\infty} \frac{d t}{t} \frac{i}{2 \sinh (\pi|\nu|)\left(8 \pi^{2} t \alpha^{\prime}\right)^{\frac{p-1}{2}} \vartheta_{1}(i \mid \nu \| i t) \eta(i t)^{d-3}} .
$$

This expression may be written by performing the modular transformation as

$$
\mathcal{A}=\sum_{k \neq 0} \int_{0}^{\infty} d s \frac{e^{-\pi s \nu^{2}}}{2 \sinh (\pi|\nu|)\left(8 \pi^{2} \alpha^{\prime}\right)^{\frac{p-1}{2}} s^{\frac{d \perp}{2}} \vartheta_{1}(|\nu| s \mid i s) \eta(i s)^{d-3}},
$$

which will be obtained as a scattering amplitude between boundary states in the next subsection.

\subsubsection{Boundary state}

The D1-brane can wrap the while Misner space, thus the winding closed strings with macroscopic length can couple to the D-brane. This fact implies that the corresponding boundary state is constructed from closed strings in the twisted sectors as well as in the untwisted sector. Since the untwisted sector is the same as in the flat space case, we concentrate on the twisted sectors. First we consider the case without flux, then the boundary state satisfies the boundary conditions for $X^{ \pm}$as

$$
\left.\left(\alpha_{n}^{ \pm}+\tilde{\alpha}_{-n}^{ \pm}\right)|D 1, w\rangle\right\rangle=0
$$

for all $n \in \mathbb{Z}$, where the label $w$ represents the winding number. In terms of oscillators, the boundary states are expressed as $(\nu=\gamma w)$

$$
|D 1, w\rangle\rangle=\exp \left(\sum_{n \geq 1} \frac{\alpha_{-n}^{+} \tilde{\alpha}_{-n}^{-}}{n+i \nu}+\sum_{n \geq 0} \frac{\alpha_{-n}^{-} \tilde{\alpha}_{-n}^{+}}{n-i \nu}\right)|0, w\rangle .
$$


We should also note that the boundary states are invariant under the discrete boost

$$
\exp (2 \pi i \gamma(J+\tilde{J}))|D 1, w\rangle\rangle=|D 1, w\rangle\rangle
$$

The full boundary states are constructed by summing over all the twisted sectors. We can introduce gauge flux on the D-brane by inserting Wilson line into the boundary state as $62{ }^{16}$

$$
W=\mathrm{P} \exp \left(-\frac{i}{2 \pi \alpha^{\prime}} \int A_{+} d X^{+}\right) .
$$

When the overlap between two boundary states for the same D-branes is computed, the Wilson lines are cancelled with each other. Therefore, the cylinder amplitude does not change by the introduction of gauge flux as before.

Using the ghost part $\left.\left|B_{g h}\right\rangle\right\rangle$ and $d$ free boson part $\left.|B(p-1)\rangle\right\rangle$ (3.39) with $(p-1)$ Neumann and $d_{\perp}$ Dirichlet boundary conditions, the full boundary state may be written as

$$
\left.\left.\left.|D p, e x t\rangle\rangle=\sum_{w} \mathcal{N}_{w}\left|B_{g h}\right\rangle\right\rangle \otimes W|D 1, w\rangle\right\rangle \otimes|B(p-1)\rangle\right\rangle
$$

with a normalization $\mathcal{N}_{w}$. Then the overlap between the boundary states is computed as

$$
\langle\langle D p, e x t|\Delta| D p, e x t\rangle\rangle=\frac{\alpha^{\prime}}{2 \pi} \int d s\left\langle\left\langle D p, e x t\left|e^{-\pi s\left(L_{0}+\tilde{L}_{0}-\frac{c}{24}\right)}\right| D p, e x t\right\rangle\right\rangle .
$$

Using the overlaps in the Misner space part ${ }^{17}$

$$
\begin{aligned}
\left\langle\left\langle D 1, w\left|e^{-\pi s\left(L_{0}+\tilde{L}_{0}-\frac{1}{6}\right)}\right| D 1, w\right\rangle\right\rangle & =\frac{i e^{-\pi s(i|\nu|(1-i|\nu|))+\frac{\pi s}{6}}}{\left(1-e^{-2 \pi i s|\nu|}\right) \prod_{n \geq 1}\left(1-e^{-2 \pi s(n+i|\nu|)}\right)\left(1-e^{-2 \pi s(n-i|\nu|)}\right)} \\
& =\frac{\eta(i s) e^{-\pi s \nu^{2}}}{\vartheta_{1}(s|\nu| \mid i s)}
\end{aligned}
$$

we can reproduce the open string partition function (3.61) if we set

$$
\mathcal{N}_{w}=\sqrt{\frac{8 \pi^{2} \alpha^{\prime}}{2 \sinh (\pi|\nu|)}} \mathcal{N}_{p} .
$$

Notice that the coefficients are different in each twisted sectors.

\subsubsection{Emission of winding closed strings}

As in the annulus amplitude for the localized D-brane, the amplitude is given by integrating over the moduli parameter and the integration leads to imaginary part. For

\footnotetext{
${ }^{16}$ We can also introduce non-trivial holonomy as $W=\exp (i \theta w)$ in $w$-th twisted sector, which also does not change the overlaps for the same D-brane. See 39] for null brane case.

${ }^{17}$ As before, we replaced $\nu$ by $|\nu|$ due to the subtlety of zero modes.
} 
our purpose, it is not useful to adopt the expression in the open string channel (3.60) since the divergence originates from the sum over the states along Regge trajectories as in the previous case. Therefore, we investigate the imaginary part in the closed string channel (3.61). The integration of $s$ encounters poles at $s=n /|\nu|$ with $n=1,2, \cdots$, and the contributions from poles are summarized as

$$
-2 \operatorname{Im} \mathcal{A}=\sum_{k \neq 0} \sum_{n=1}^{\infty} \frac{(-1)^{n+1}}{2|\nu| \sinh (\pi|\nu|)\left(8 \pi^{2} \alpha^{\prime}\right)^{\frac{p-1}{2}}}\left(\frac{|\nu|}{n}\right)^{\frac{d_{\perp}}{2}} \sum_{\text {states }} e^{-2 \pi n \frac{N-\frac{d}{24}}{\nu \mid}-\pi n|\nu|} .
$$

Again the imaginary part diverges for $n /|\nu|<1$ due to the Hagedorn behavior $\rho_{N} \sim e^{2 \pi N}$.

Following the previous analysis, we identify the origin of the imaginary part. Using the identity (2.39) with $T=\pi s|\nu|$, we can rewrite the partition function (3.61) as

$$
\begin{aligned}
\mathcal{A} & =\sum_{k \neq 0} \frac{\alpha^{\prime}}{2 \pi} \int_{0}^{\infty} d s \int_{-\infty}^{\infty} d\left(\omega^{2}\right) \int \frac{d^{d_{\perp}} k_{\perp}}{(2 \pi)^{d_{\perp}}} \sum_{\text {states }} \mathcal{N}_{w}^{2} \rho\left(\omega^{2}\right) e^{-2 \pi s\left(\omega^{2}|\nu|+\frac{\alpha^{\prime}}{2} \vec{k}_{\perp}^{2}+N-\frac{d}{24}+\frac{\nu^{2}}{2}\right)} \\
& =\sum_{k \neq 0} \frac{\alpha^{\prime}}{2 \pi} \int_{-\infty}^{\infty} d\left(\omega^{2}\right) \int \frac{d^{d_{\perp}} k_{\perp}}{(2 \pi)^{d_{\perp}}} \sum_{\text {states }} \frac{\mathcal{N}_{w}^{2} \rho\left(\omega^{2}\right)}{2 \pi\left(\omega^{2}|\nu|+\frac{\alpha^{\prime}}{2} \vec{k}_{\perp}^{2}+N-\frac{d}{24}+\frac{\nu^{2}}{2}\right)}
\end{aligned}
$$

where we denote $\vec{k}_{\perp}$ as the momenta for directions transverse to the D-brane. The imaginary part arises from the poles of the closed string propagator $L_{0}+\tilde{L}_{0}-\frac{d}{12}=0$, therefore we identify that the imaginary part (3.70) is due to the closed string emission (see fig. 2). Note that the closed strings couple to D-branes only if the modes satisfy the boundary condition (3.62). In particular, the quasi zero modes satisfy $\alpha_{0}^{ \pm}+\tilde{\alpha}_{0}^{ \pm}=0$, which says that only strings (2.10) localized in the whisker regions are emitted from the D-branes. ${ }^{18}$

The emission of closed strings from D-branes was first discussed in 63, 64] in the configuration of decaying D-branes with rolling tachyon [65, 66]. In the context, the Dbrane disappears as the tachyon condenses, and the energy is taken out by emitted closed strings. Similar observation was given in 41, 42, 43, 44, 45, where a D-brane is falling into a stack of NS5-branes [40]. The D-brane will form a bound state with NS5-branes, and the closed strings carry out the generated energy. The above examples are dynamics in static backgrounds, but our case is in a time-dependent space-time. Thus we expect that the winding strings are emitted from D-branes due to the time-dependent effects. Actually, the time-dependence enters through the orbifold action (2.1), therefore it is natural that the only closed strings in the twisted sectors feel the time-dependence and are emitted from the D-brane. ${ }^{19}$ We should remark that the emission rate of winding

\footnotetext{
${ }^{18}$ This might be related to the conjecture [24] that the condensation of strings localized in the regions with CTC protects the chronology, which is supposed to be the string version of chronology protection conjecture 25.

${ }^{19}$ As mentioned before, the untwisted sector is the same as the flat space case, thus there is no closed string emission from the sector.
} 
strings diverges for $n /|\nu|<1$. Since the closed strings are emitted, the background itself may largely change due to the emission.

Finally, we check that the previous expression (3.70) can be reproduced by collecting poles of propagator as

$$
-2 \operatorname{Im} \mathcal{A}=\sum_{k \neq 0} \int \frac{d^{d_{\perp}} k_{\perp}}{(2 \pi)^{d_{\perp}}} \frac{\alpha^{\prime}}{2 \pi} \frac{\mathcal{N}_{w}^{2}}{|\nu|} \sum_{n=1}^{\infty} \sum_{\text {states }}(-1)^{n+1} e^{-\frac{2 \pi n}{|\nu|}\left(\frac{\alpha^{\prime}}{2} \vec{k}_{\perp}^{2}+N-\frac{d}{24}+\frac{\nu^{2}}{2}\right)} .
$$

Here we have used the identity (3.45).

\section{Superstrings and D-branes in Misner space}

The bosonic string theory includes tachyonic states with no excitation of oscillator modes, therefore the system is badly unstable. In order to see that the instability of Dbranes is not related to the tachyonic modes, we consider type IIA or type IIB superstring theory. In this section, we study one-loop amplitude to obtain tachyon free results. We only adopt the oscillator formalism, but the same results should be obtained in the path integral formalism.

\subsection{Torus amplitude}

We consider type IIA or type IIB superstrings on the manifold $\left[\mathbb{R}^{1,1} / \Gamma\right] \times \mathbb{R}^{8}$ that is a product of Misner space and 8 dimensional flat space. In the $w$-th twisted sector, fermions have to obey the conditions

$$
\psi^{ \pm}(\tau, \sigma+2 \pi)=-(-1)^{A} e^{ \pm 2 \pi \nu} \psi^{ \pm}(\tau, \sigma), \quad \tilde{\psi}^{ \pm}(\tau, \sigma+2 \pi)=-(-1)^{A} e^{ \pm 2 \pi \nu} \tilde{\psi}^{ \pm}(\tau, \sigma)
$$

where $A=0$ for NS-sector and $A=1$ for R-sector. The mode expansions can be given then

$$
\psi^{ \pm}=\sum_{r} b_{-r}^{ \pm} e^{-i(r \pm i \nu)(\tau+\sigma)}, \quad \quad \tilde{\psi}^{ \pm}=\sum_{r} \tilde{b}_{-r}^{ \pm} e^{-i(r \mp i \nu)(\tau-\sigma)}
$$

with $r \in \mathbb{Z}+(A+1) / 2$. The oscillators satisfy the anti-commutation relations

$$
\left\{b_{r}^{ \pm}, b_{s}^{\mp}\right\}=-\delta_{r+s}, \quad\left\{\tilde{b}_{r}^{ \pm}, \tilde{b}_{s}^{\mp}\right\}=-\delta_{r+s} .
$$

The Virasoro generators are

$$
\begin{aligned}
& L_{0}^{f}=-\frac{1}{2} \nu^{2}-\sum_{r \geq 1 / 2}(r-i \nu) b_{-r}^{+} b_{r}^{-}-\sum_{r \geq 1 / 2}(r+i \nu) b_{-r}^{-} b_{r}^{+} \\
& \tilde{L}_{0}^{f}=-\frac{1}{2} \nu^{2}-\sum_{r \geq 1 / 2}(r+i \nu) \tilde{b}_{-r}^{+} \tilde{b}_{r}^{-}-\sum_{r \geq 1 / 2}(r-i \nu) \tilde{b}_{-r}^{-} \tilde{b}_{r}^{+}
\end{aligned}
$$


for NSNS-sector and

$$
\begin{aligned}
& L_{0}^{f}=\frac{1}{8}-\frac{i \nu(1-i \nu)}{2}-\sum_{r \geq 1}(r-i \nu) b_{-r}^{+} b_{r}^{-}-\sum_{r \geq 0}(r+i \nu) b_{-r}^{-} b_{r}^{+}, \\
& \tilde{L}_{0}^{f}=\frac{1}{8}-\frac{i \nu(1-i \nu)}{2}-\sum_{r \geq 0}(r+i \nu) \tilde{b}_{-r}^{+} \tilde{b}_{r}^{-}-\sum_{r \geq 1}(r-i \nu) \tilde{b}_{-r}^{-} \tilde{b}_{r}^{+}
\end{aligned}
$$

for RR-sector. The Virasoro generators for NSR and RNS-sectors are given in a similar way.

In order to obtain the full partition function, we add flat 8 dimension and ghost part, and assign the GSO projection necessary to project out the unphysical states. Denoting $F, \tilde{F}$ as the worldsheet fermion number operators, the partition function can be computed as

$$
\begin{aligned}
\mathcal{T}=\int_{\mathcal{F}} \frac{d^{2} \tau}{\tau_{2}} \sum_{w, k} & {\left[\operatorname{Tr}_{N S N S}^{w}\left(\frac{1}{4}\left(1+(-1)^{F}\right)\left(1+(-1)^{\tilde{F}}\right) g^{k} q^{L_{0}} \bar{q}^{\tilde{L}_{0}}\right)\right.} \\
& -\operatorname{Tr}_{N S R}^{w}\left(\frac{1}{4}\left(1+(-1)^{F}\right)\left(1 \mp(-1)^{\tilde{F}}\right) g^{k} q^{L_{0}} \bar{q}^{\tilde{L}_{0}}\right) \\
& -\operatorname{Tr}_{R N S}^{w}\left(\frac{1}{4}\left(1+(-1)^{F}\right)\left(1+(-1)^{\tilde{F}}\right) g^{k} q^{L_{0}} \bar{q}^{\tilde{L}_{0}}\right) \\
& \left.+\operatorname{Tr}_{R R}^{w}\left(\frac{1}{4}\left(1+(-1)^{F}\right)\left(1 \mp(-1)^{\tilde{F}}\right) g^{k} q^{L_{0}} \bar{q}^{\tilde{L}_{0}}\right)\right],
\end{aligned}
$$

where we use different GSO projections for type IIA and type IIB. Namely, we assign for type IIA and + for type IIB. The trace $\operatorname{Tr}^{w}$ is taken in the $w$-th twisted sector and the boost operator $g=\exp (2 \pi \gamma i(J+\tilde{J}))$ is defined by

$$
i J=-\sum_{r \geq \frac{1}{2}} b_{-r}^{+} b_{r}^{-}+\sum_{r \geq \frac{1}{2}} b_{-r}^{-} b_{r}^{+}, \quad i \tilde{J}=-\sum_{r \geq \frac{1}{2}} \tilde{b}_{-r}^{+} \tilde{b}_{r}^{-}+\sum_{r \geq \frac{1}{2}} \tilde{b}_{-r}^{-} \tilde{b}_{r}^{+}
$$

for NSNS-sector,

$$
i J=-\sum_{n \geq 1} b_{-n}^{+} b_{n}^{-}+\sum_{n \geq 0} b_{-n}^{-} b_{n}^{+}, \quad i \tilde{J}=-\sum_{n \geq 0} \tilde{b}_{-n}^{+} \tilde{b}_{n}^{-}+\sum_{n \geq 1} \tilde{b}_{-n}^{-} \tilde{b}_{n}^{+}
$$

for RR-sector, and similarly for NSR and RNS-sectors. This one-loop amplitude can be evaluated as 4 ]

$$
\mathcal{T}=\int_{\mathcal{F}} \frac{d^{2} \tau}{16 \pi^{2} \tau_{2}^{2} \alpha^{\prime}} \sum_{k, w} \frac{\left|\vartheta_{3}(y \mid \tau) \vartheta_{3}(0 \mid \tau)^{3}-\vartheta_{4}(y \mid \tau) \vartheta_{4}(0 \mid \tau)^{3}-\vartheta_{2}(y \mid \tau) \vartheta_{2}(0 \mid \tau)^{3}\right|^{2}}{\left(4 \pi^{2} \tau_{2} \alpha^{\prime}\right)^{3}\left|\vartheta_{1}(y \mid \tau) \eta(\tau)^{9}\right|^{2}}
$$

with $y=i \gamma(w \tau+k)$. This can be expressed as

$$
\mathcal{T}=\int_{\mathcal{F}} \frac{d^{2} \tau}{4 \pi^{2} \tau_{2}^{2} \alpha^{\prime}} \sum_{k, w} \frac{\left|\vartheta_{1}(y / 2 \mid \tau)^{4}\right|^{2}}{\left(4 \pi^{2} \tau_{2} \alpha^{\prime}\right)^{3}\left|\vartheta_{1}(y \mid \tau) \eta(\tau)^{9}\right|^{2}}
$$


using the identity

$$
\vartheta_{3}(y \mid \tau) \vartheta_{3}(0 \mid \tau)^{3}-\vartheta_{4}(y \mid \tau) \vartheta_{4}(0 \mid \tau)^{3}-\vartheta_{2}(y \mid \tau) \vartheta_{2}(0 \mid \tau)^{3}=2 \vartheta_{1}(y / 2 \mid \tau)^{4} .
$$

The one-loop amplitude does not vanish because the supersymmetry is broken though the boundary conditions (2.4) and (4.1). Notice that tachyonic state in NSNS-sector, which is apparent signal of background instability, is projected out by the GSO projection.

Before moving to the open string sector, we should comment on the case with antiperiodic boundary condition for fermions. Namely, we can consistently assign

$$
\begin{aligned}
& \psi^{ \pm}(\tau, \sigma+2 \pi)=-(-1)^{A+w} e^{ \pm 2 \pi \nu} \psi^{ \pm}(\tau, \sigma), \\
& \tilde{\psi}^{ \pm}(\tau, \sigma+2 \pi)=-(-1)^{A+w} e^{ \pm 2 \pi \nu} \tilde{\psi}^{ \pm}(\tau, \sigma) .
\end{aligned}
$$

The action of GSO projection is not changed for even $w$ but opposite for odd $w$. Therefore, the tachyonic modes appear in the odd $w$-th twisted sector, which will be a signal of background instability. The condensation of twisted closed tachyons is discussed in [24, 28] using the similarity to the condensation of localized tachyon in Euclidean orbifolds $\mathbb{C} / \mathbb{Z}_{N}$ 67. Thus, the case with anti-periodic fermions would be important, if we consider the condensation of winding closed tachyons.

\subsection{Localized D-brane}

We first consider $\mathrm{D} p$-brane localized in the Misner space part. ${ }^{20}$ More precisely, we consider the D-brane at $e^{2 \pi a} x^{+}+b=e^{-2 \pi a} x^{-}-b$ and their images of orbifold action in the covering space. The boundary conditions for fermions in Misner space are given by

$$
\begin{aligned}
& \psi^{ \pm}(\tau, \sigma=0)=e^{\mp 4 \pi\left(a+\gamma k_{1}\right)} \tilde{\psi}^{\mp}(\tau, \sigma=0), \\
& \psi^{ \pm}(\tau, \sigma=\pi)=-(-1)^{A} e^{\mp 4 \pi\left(a+\gamma k_{2}\right)} \tilde{\psi}^{\mp}(\tau, \sigma=\pi),
\end{aligned}
$$

where $A=0$ for NS sector and $A=1$ for R-sector. Then, the mode expansions read

$$
\psi^{ \pm}=\sum_{r} b_{-r}^{ \pm} e^{\mp 2 \pi\left(a+\gamma k_{1}\right)-i(r \pm i \nu)(\tau+\sigma)}, \quad \tilde{\psi}^{ \pm}=\sum_{r} b_{-r}^{\mp} e^{\mp 2 \pi\left(a+\gamma k_{1}\right)-i(r \mp i \nu)(\tau-\sigma)},
$$

with $\left\{b_{r}^{+}, b_{s}^{-}\right\}=-\delta_{r+s}$. Here $\nu=2 \gamma\left(k_{1}-k_{2}\right)$ and $r \in \mathbb{Z}+(A+1) / 2$. The Virasoro generators are

$$
L_{0}^{f}=-\frac{1}{2} \nu^{2}-\sum_{r \geq 1 / 2}(r-i \nu) b_{-r}^{+} b_{r}^{-}-\sum_{r \geq 1 / 2}(r+i \nu) b_{-r}^{-} b_{r}^{+}
$$

for NS-sector and

$$
L_{0}^{f}=\frac{1}{8}-\frac{i \nu(1-i \nu)}{2}-\sum_{r \geq 1}(r-i \nu) b_{-r}^{+} b_{r}^{-}-\sum_{r \geq 0}(r+i \nu) b_{-r}^{-} b_{r}^{+}
$$

\footnotetext{
${ }^{20}$ We set $p$ even for type IIA and odd for type IIB as usual.
} 
for R-sector. For other 8 coordinates, we again assign the Neumann boundary condition for $p$ coordinates and the Dirichlet boundary condition for $8-p$ coordinates. Then, the partition function is ${ }^{21}$

$$
\mathcal{A}=\int_{0}^{\infty} \frac{d t}{2 t} \frac{\vartheta_{3}(|\nu| t \mid i t) \vartheta_{3}(0 \mid i t)^{3}-\vartheta_{4}(|\nu| t \mid i t) \vartheta_{4}(0 \mid i t)^{3}-\vartheta_{2}(|\nu| t \mid i t) \vartheta_{2}(0 \mid i t)^{3}}{\left(8 \pi^{2} t \alpha^{\prime}\right)^{\frac{p}{2}} \vartheta_{1}(|\nu| t \mid i t) \eta(i t)^{9}}
$$

or by modular transformation $(s=1 / t)$

$$
\mathcal{A}=\int_{0}^{\infty} \frac{i d s}{2} \frac{\vartheta_{3}(i|\nu| \mid i t) \vartheta_{3}(0 \mid i s)^{3}-\vartheta_{4}(i \mid \nu \| i s) \vartheta_{4}(0 \mid i s)^{3}-\vartheta_{2}(i \mid \nu \| i s) \vartheta_{2}(0 \mid i s)^{3}}{\left(8 \pi^{2} \alpha^{\prime}\right)^{\frac{p}{2}} s^{\frac{8-p}{2}} \vartheta_{1}(i \mid \nu \| i s) \eta(i s)^{9}} .
$$

This partition function should be reproduced as a scattering amplitude between boundary states.

As in the bosonic case, we first construct boundary state for the original D-brane and generate boundary states for the image branes by utilizing the orbifold action. For the $\mathrm{D} p$-brane at $e^{2 \pi a} x^{+}+b=e^{-2 \pi a} x^{-}-b$, the boundary state $\left.|D p, \eta\rangle\right\rangle_{P}(P=N S N S$ or $R R)$ satisfies the conditions

$$
\begin{aligned}
& \left.\left(e^{ \pm 2 \pi a} b_{-r}^{ \pm}+i \eta e^{\mp 2 \pi a} \tilde{b}_{r}^{ \pm}\right)|D p, \eta\rangle\right\rangle_{P}=0 \\
& \left.\left(b_{-r}^{\alpha}+i \eta \tilde{b}_{r}^{\alpha}\right)|D p, \eta\rangle\right\rangle_{P}=0 \\
& \left.\left(b_{-r}^{I}-i \eta \tilde{b}_{r}^{I}\right)|D p, \eta\rangle\right\rangle_{P}=0 .
\end{aligned}
$$

Here we used indices $2 \leq \alpha \leq p$ for directions parallel to $\mathrm{D} p$-brane and $p+1 \leq I \leq d+1$ for transverse directions. We also use $r \in \mathbb{Z}+\frac{1}{2}$ for $P=N S N S$ and $r \in \mathbb{Z}$ for $P=R R$, and $\eta= \pm 1$ representing the spin structure. The image branes are generated as

$$
\left.|D p, k, \eta\rangle\rangle_{P}=e^{2 \pi i \gamma k(J+\tilde{J})}|D p, \eta\rangle\right\rangle_{P}
$$

where the boost operator is defined as (4.7) and (4.8). Then the fermionic parts of the boundary state are explicitly written as

$$
\begin{aligned}
|D p, k, \eta\rangle\rangle_{N S N S} & =e^{i \eta \sum_{r>0}\left(e^{4 \pi \kappa} b_{-r}^{+} \tilde{b}_{-r}^{+}+e^{-4 \pi \kappa} b_{-r}^{-} \tilde{b}_{-r}^{-}-b_{-r}^{\alpha} \tilde{b}_{-r}^{\alpha}+b_{-r}^{I} \tilde{b}_{-r}^{I}\right)}|0\rangle \\
|D p, k, \eta\rangle\rangle_{R R} & =e^{i \eta \sum_{n>0}\left(e^{4 \pi \kappa} b_{-n}^{+} \tilde{b}_{-n}^{+}+e^{-4 \pi \kappa} b_{-n}^{-} \tilde{b}_{-n}^{-}-b_{-n}^{\alpha} \tilde{b}_{-n}^{\alpha}+b_{-n}^{I} \tilde{b}_{-n}^{I}\right)}|k, \eta\rangle_{R R}^{(0)}
\end{aligned}
$$

with $\kappa=a+\gamma k$. We use the normalization of $\mathrm{RR}$ vacuum states $\mathrm{as}^{22}$

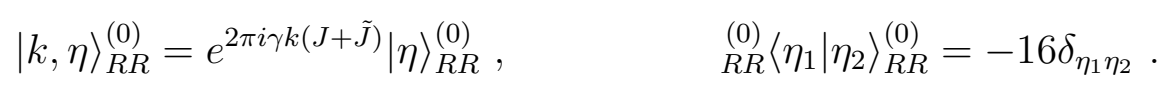

\footnotetext{
${ }^{21}$ Here we replaced $\nu$ by $|\nu|$ from the same reason as before.

${ }^{22}$ See, e.g., 68, 69] for the explicit form and other properties of the RR vacuum states.
} 
The boundary state also includes ghost part and bosonic part with $d=8$ given above. The GSO invariant combination is

$$
\begin{aligned}
|D p, l o c\rangle\rangle & \left.\left.=\frac{1}{2}(|D p, l o c,+\rangle\rangle_{N S N S}-|D p, l o c,-\rangle\right\rangle_{N S N S}\right) \\
& \left.\left.+\frac{1}{2}(|D p, l o c,+\rangle\rangle_{R R}+|D p, l o c,-\rangle\right\rangle_{R R}\right)
\end{aligned}
$$

where the sign in front of the RR sector is changed from + to - for anti-D-brane.

We would like to show the scattering amplitude between the boundary states reproduces the amplitude (4.18). A non-trivial contribution comes from the zero mode part of RR-sector. For the part, the boost operator is written as

$$
i J+i \tilde{J}=b_{0}^{-} b_{0}^{+}-\tilde{b}_{0}^{+} \tilde{b}_{0}^{-}=d_{+}^{t} d_{-}^{x}+d_{-}^{t} d_{+}^{x}
$$

where we denote

$$
d_{ \pm}^{t}=\frac{1}{2}\left[\left(b_{0}^{+}+b_{0}^{-}\right) \pm i\left(\tilde{b}_{0}^{+}+\tilde{b}_{0}^{-}\right)\right], \quad d_{ \pm}^{x}=\frac{1}{2}\left[\left(b_{0}^{+}-b_{0}^{-}\right) \pm i\left(\tilde{b}_{0}^{+}-\tilde{b}_{0}^{-}\right)\right] .
$$

Note that the new operators have the following properties as

$$
\left\{d_{+}^{i}, d_{-}^{j}\right\}=\delta^{i j}, \quad d_{\eta}^{t}|\eta\rangle_{R R}^{(0)}=0, \quad d_{-\eta}^{x}|\eta\rangle_{R R}^{(0)}=0 .
$$

Then, we obtain by following [57] as

$$
\left.\left|k, \eta>_{R R}^{(0)}=e^{2 \pi i \gamma k(J+\tilde{J})}\right| \eta\right\rangle_{R R}^{(0)}=\left[\cosh (2 \pi \gamma k)+\sinh (2 \pi \gamma k) d_{-\eta}^{t} d_{\eta}^{x}\right]|\eta\rangle_{R R}^{(0)}
$$

and hence

$$
{ }_{R R}^{(0)}\left\langle k_{1}, \eta \mid k_{2}, \eta\right\rangle_{R R}^{(0)}=-16 \cosh \left(2 \pi \gamma\left|k_{1}-k_{2}\right|\right) .
$$

Combining this result and contributions from other fermionic modes, bosonic modes and ghost modes, we can show that the overlap reproduces the partition function (4.18).

Finally let us evaluate the imaginary part of the one-loop amplitude (4.17). As in the bosonic case, the poles in the integration over $t$ leads to the imaginary part as

$$
-2 \operatorname{Im} \mathcal{A}=\sum_{k, k^{\prime}} \sum_{n=1}^{\infty} \sum_{\text {states }} \frac{(-1)^{(n+1)(A+1)}}{|\nu|\left(8 \pi^{2} \alpha^{\prime}\right)^{\frac{p}{2}}}\left(\frac{|\nu|}{n}\right)^{\frac{p}{2}+1} e^{-2 \pi n \frac{N}{\nu \mid}},
$$

where $A=0$ for bosons (NS-sector) and $A=1$ for fermions (R-sector). Thus, in the supersymmetric case, the sign depends on whether the state is bosonic or fermionic, and the zero point energy shift by $\frac{1}{2}|\nu|^{2}$ disappears. Notice that tachyonic state is projected out from the spectrum of pair created open string $N$, which includes fermionic sector as well. Therefore, we can say that the instability due to the open string pair creation is not related to the tachyonic mode. 


\subsection{Wrapping D-brane}

Next let us generalize D $p$-brane wrapping the whole Misner space to superstring case. Just like the bosonic case, we introduce a constant gauge flux $F^{+-}=f$ on D-brane in the covering space. We adopt for Misner space part fermions with boundary conditions

$$
\begin{array}{ll}
\psi^{ \pm}-\tilde{\psi}^{ \pm}= \pm f\left(\psi^{ \pm}+\tilde{\psi}^{ \pm}\right) & \text {at } \sigma=0, \\
\psi^{ \pm}-\tilde{\psi}^{ \pm}=\mp(-1)^{A} f\left(\psi^{ \pm}+\tilde{\psi}^{ \pm}\right) & \text {at } \sigma=\pi,
\end{array}
$$

where $A=0$ and $A=1$ correspond to NS-sector and R-sector, respectively. Therefore, we have the mode expansions

$$
\psi^{ \pm}=\sum_{r} b_{-r}^{ \pm} e^{-i r(\tau+\sigma) \pm \operatorname{arctanh} f}, \quad \tilde{\psi}^{ \pm}=\sum_{r} b_{-r}^{ \pm} e^{-i r(\tau-\sigma) \mp \operatorname{arctanh} f}
$$

with $r \in \mathbb{Z}+(A+1) / 2$ and the Virasoro generator

$$
L_{0}^{f}=c(A)-\sum_{r>0} r b_{-r}^{+} b_{r}^{-}-\sum_{r>0} r b_{-r}^{-} b_{r}^{+}
$$

with $c(0)=0$ and $c(1)=\frac{1}{8}$. Here the oscillators satisfy $\left\{b_{r}^{ \pm}, b_{s}^{\mp}\right\}=-\delta_{r+s}$. We will use the projection operator defined as

$$
P=\sum_{k} e^{2 \pi i \gamma k \hat{J}}, \quad \quad e^{2 \pi i \gamma \hat{J}} \psi^{ \pm}=e^{ \pm 2 \pi \gamma} \psi^{ \pm}
$$

For other 8 coordinates, we assign the Neumann boundary condition for $p-1$ coordinates and the Dirichlet boundary condition for $9-p$ coordinates. Taking into account the GSO projection, the partition function can be written as

$$
\mathcal{A}(t)=\operatorname{Tr}_{\mathcal{H}_{\mathrm{NS}}} P \frac{1}{2}\left(1+(-1)^{F}\right) e^{-2 \pi t L_{0}}-\operatorname{Tr}_{\mathcal{H}_{\mathrm{R}}} P \frac{1}{2}\left(1 \pm(-1)^{F}\right) e^{-2 \pi t L_{0}}
$$

Including ghost parts, we obtain

$$
\mathcal{A}=\int_{0}^{\infty} \frac{i d t}{2 t} \frac{\vartheta_{4}(i|k| \gamma \mid i t) \vartheta_{4}(0 \mid i t)^{3}-\vartheta_{3}(i|k| \gamma \mid i t) \vartheta_{3}(0 \mid i t)^{3}-\vartheta_{2}(i|k| \gamma \mid i t) \vartheta_{2}(0 \mid i t)^{3}}{2 \sinh (\pi|k| \gamma)\left(8 \pi^{2} t \alpha^{\prime}\right)^{\frac{p-1}{2}} \vartheta_{1}(i|k| \gamma \mid i t) \eta(i t)^{9}}
$$

Using the modular transformation, we could write as

$$
\mathcal{A}=\int_{0}^{\infty} \frac{d s}{2} \frac{\vartheta_{3}(|k| \gamma s \mid i s) \vartheta_{3}(0 \mid i s)^{3}-\vartheta_{4}(|k| \gamma s \mid i s) \vartheta_{4}(0 \mid i s)^{3}-\vartheta_{2}(|k| \gamma s \mid i s) \vartheta_{2}(0 \mid i s)^{3}}{2 \sinh (\pi|k| \gamma)\left(8 \pi^{2} \alpha^{\prime}\right)^{\frac{p-1}{2}} s^{\frac{9-p}{2}} \vartheta_{1}(|k| \gamma s \mid i s) \eta(i s)^{9}}
$$

which should be reproduced by an overlap between boundary states.

As in the bosonic case, we construct boundary states only in the twisted sectors. The boundary states can be written as the product

$$
\left.\left.\left.|D p, e x t, \eta\rangle\rangle_{P}=\sum_{w} \mathcal{N}_{w}\left|B_{g h .}, \eta\right\rangle\right\rangle_{P} \otimes W|D 1, w, \eta\rangle\right\rangle_{P} \otimes|B(p-1), \eta\rangle\right\rangle_{P}
$$


where $P=N S N S$ or $R R$ and $\mathcal{N}_{w}$ is (3.69) with $d=8$. The Wilson line also includes fermionic part as

$$
W=\mathrm{P} \exp \left[-\frac{i}{2 \pi \alpha^{\prime}} \int_{0}^{2 \pi} d \sigma\left(A_{\mu} \partial_{\sigma} X^{\mu}-\frac{i}{2} F_{\mu \nu} \theta^{\mu} \theta^{\nu}\right)\right], \quad \theta^{\mu}=\psi^{\mu}-i \eta \tilde{\psi}^{\mu},
$$

though it gives no contribution to the overlaps for the same D-brane. The boundary states given above satisfy the boundary conditions

$$
\begin{aligned}
& \left.\left(b_{-r}^{ \pm}+i \eta \tilde{b}_{r}^{ \pm}\right)|D 1, w, \eta\rangle\right\rangle_{P}=0 \\
& \left.\left(b_{-r}^{\alpha}+i \eta \tilde{b}_{r}^{\alpha}\right)|B(p-1), \eta\rangle\right\rangle_{P}=0 \\
& \left.\left(b_{-r}^{I}-i \eta \tilde{b}_{r}^{I}\right)|B(p-1), w, \eta\rangle\right\rangle_{P}=0
\end{aligned}
$$

where $2 \leq \alpha \leq p-1$ for directions tangent to D-brane and $p \leq I \leq d+1$ for normal directions. Picking up the fermionic part, they are expressed as

$$
\begin{gathered}
|D 1, w, \eta\rangle\rangle_{N S N S}=e^{i \eta \sum_{n \geq 1}\left(b_{-n+1 / 2}^{+} \tilde{b}_{-n+1 / 2}^{-}+b_{-n+1 / 2}^{-} \tilde{b}_{-n+1 / 2}^{+}\right)}|0\rangle, \\
|D 1, w, \eta\rangle\rangle_{R R}=e^{i \eta\left(\sum_{n \geq 1} b_{-n}^{+} \tilde{b}_{-n}^{-}+\sum_{n \geq 0} b_{-n}^{-} \tilde{b}_{-n}^{+}\right)}|0\rangle
\end{gathered}
$$

for Misner part and

$$
\begin{gathered}
|B(p-1), \eta\rangle\rangle_{N S N S}=e^{-i \eta \sum_{n \geq 1}\left(b_{-n+1 / 2}^{\alpha} \tilde{b}_{-n+1 / 2}^{\alpha}-b_{-n+1 / 2}^{I} \tilde{b}_{-n+1 / 2}^{I}\right)}|0\rangle, \\
|D 1, w, \eta\rangle\rangle_{R R}=e^{-i \eta\left(\sum_{n \geq 1} b_{-n}^{\alpha} \tilde{b}_{-n}^{\alpha}-\sum_{n \geq 1} b_{-n}^{I} \tilde{b}_{-n}^{I}\right)}|\eta\rangle_{R R}^{(0)}
\end{gathered}
$$

for the other 8 dimension. The normalization of $\mathrm{RR}$ vacuum states is set as ${ }_{R R}^{(0)}\left\langle\eta_{1} \mid \eta_{2}\right\rangle_{R R}^{(0)}=$ $-8 \delta_{\eta_{1} \eta_{2}}$. The GSO invariant combination is given by

$$
\begin{aligned}
|D p, e x t\rangle\rangle & \left.\left.=\frac{1}{2}(|D p, e x t,+\rangle\rangle_{N S N S}-|D p, e x t,-\rangle\right\rangle_{N S N S}\right) \\
& \left.\left.+\frac{1}{2}(|D p, e x t,+\rangle\rangle_{R R}+|D p, e x t,-\rangle\right\rangle_{R R}\right)
\end{aligned}
$$

Computing the overlaps between the boundary states, we reproduce (4.36).

The imaginary part of the cylinder amplitude arises when integrating (4.36) by the moduli parameter $s$. In order to avoid divergence, we shift the contour and then pick up the poles as

$$
-2 \operatorname{Im} \mathcal{A}=\sum_{w \neq 0} \sum_{n=1}^{\infty} \sum_{\text {states }} \frac{(-1)^{(n+1)(A+1)}}{2|\nu| \sinh (\pi|\nu|)\left(8 \pi^{2} \alpha^{\prime}\right)^{\frac{p-1}{2}}}\left(\frac{|\nu|}{n}\right)^{\frac{9-p}{2}} e^{-2 \pi n \frac{N}{|\nu|}},
$$

which corresponds to the rate of winding string emission as discussed in the bosonic case. We can see that there are no emission of tachyonic modes and no zero point energy shift. 


\section{Correlation functions}

As shown in 6, 9, 18, the closed string $2 \rightarrow 2$ amplitude in the untwisted sector diverges due to the graviton exchange near the big crunch/big bang singularity, which was interpreted as the signal of large back reaction. Since there is no gravity mode in open string spectrum, it is natural to expect that the divergence becomes milder for open string $2 \rightarrow 2$ scattering on wrapping D-brane, which will be examined in this section. The gauge flux on D-branes induces the (time-dependent) non-commutativity, so it is quite interesting to see if the singular behavior is improved by the non-commutativity. For definiteness, we consider $\mathrm{D} p$-brane wrapping the Misner space part in the critical bosonic string theory. Moreover, we introduce gauge flux only on the Misner space part. The generalization to the superstring case may be done by following [21].

\subsection{Wave function and two point function}

Wave function can be realized as a superposition of plane waves in the covering space as [4, 18$]^{23}$

$$
\Psi_{m, l}=\sqrt{\frac{m}{2 \pi}} \int d w e^{\frac{i m}{\sqrt{2}}\left(X^{-} e^{-w}+X^{+} e^{w}\right)+i w l+i \vec{k} \cdot \vec{X}}
$$

with $m \geq 0, l \in \mathbb{Z}$. We can easily see that the wave function is invariant under the discrete boost (2.1). Using the fact that the shift $p^{ \pm} \rightarrow e^{ \pm a} p^{ \pm}$gives only a phase factor, the momenta satisfying the on-shell condition $2 p^{+} p^{-}=m^{2}$ have been set as $p^{ \pm}=m / \sqrt{2}$. This wave function represents an excitation over the adiabatic vacuum [0], which is defined utilizing the vacuum in the Minkowski space. This means that the wave function with $p^{ \pm}>0$ represents in-coming wave. We have denoted $\vec{k}$ as momenta for the other $(p-1)$ directions parallel to the $\mathrm{D} p$-branes.

As mentioned above, we would like to introduce gauge flux on the D-brane. In the covering space, we condense constant gauge field strength, which induces non-commutativity 55

$$
\left[x^{+}, x^{-}\right]=i \Theta^{+-}(\equiv i \theta)
$$

In the presence of non-zero $\Theta^{i j}$, the correlation functions for plane waves include nontrivial phase factor as 55

$$
\left\langle\prod_{n} e^{i k_{i}^{n} X^{i}}\left(\tau_{n}\right)\right\rangle=e^{-\frac{i}{2} \sum_{n>m} k_{i}^{n} \Theta^{i j} k_{j}^{m} \epsilon\left(\tau_{n}-\tau_{m}\right)} \delta\left(\sum_{n} k^{n}\right),
$$

where $\epsilon\left(\tau_{n}-\tau_{m}\right)=1$ for $\tau_{n}>\tau_{m}$ and $\epsilon\left(\tau_{n}-\tau_{m}\right)=-1$ for $\tau_{n}<\tau_{m}$. Using this formula,

\footnotetext{
${ }^{23}$ In this section, we set $\gamma=1$ for simplicity.
} 
we can compute two point functions of (5.1) as $\left(\tau_{1}>\tau_{2}\right)$

$$
\begin{aligned}
\left\langle\Psi_{m_{2}, l_{2}}^{*} \Psi_{m_{1}, l_{1}}\right\rangle & =\frac{2 \sqrt{m_{1} m_{2}}}{\pi} \int d w_{+} e^{i\left(l_{1}-l_{2}\right) w_{+}} \int d w_{-} e^{i\left(l_{1}+l_{2}\right) w_{-}} e^{\frac{i}{4} \theta m_{1} m_{2}\left(e^{2 w_{-}}-e^{-2 w_{-}}\right)} \\
& \times \delta\left(m_{1} e^{-w_{-}}-m_{2} e^{w_{-}}\right) \delta\left(m_{1} e^{w_{-}}-m_{2} e^{-w_{-}}\right) \delta^{(p-1)}\left(\vec{k}_{1}-\vec{k}_{2}\right)
\end{aligned}
$$

where we have defined $w_{ \pm}=\frac{1}{2}\left(w_{1} \pm w_{2}\right)$. This expression reduces to

$$
\left\langle\Psi_{m_{2}, l_{2}}^{*} \Psi_{m_{1}, l_{1}}\right\rangle=\delta_{l_{1} l_{2}} \delta\left(m_{1}-m_{2}\right) \delta^{(p-1)}\left(\vec{k}_{1}-\vec{k}_{2}\right)
$$

where the normalization has been set by the definition of the wave function (5.1). Note that there is no phase shift in the two point function as in the case with a constant $\Theta^{i j}$. The two point function in the closed string case can be also computed using (5.3) with $\Theta^{i j}=0$, thus giving the same result as in the open string one.

\subsection{Three point function}

It was shown in [18] that the three point function for closed strings does not show any singular behavior since the main contribution comes from the asymptotic regions. We anticipate that the similar conclusion would be drawn also in open string case, but the three point function may include non-trivial phase factor due to the non-trivial flux. Using (15.3) , the three point function can be computed as $\left(\tau_{1}>\tau_{2}>\tau_{3}\right)$

$$
\begin{aligned}
& \left\langle\Psi_{m_{1}, l_{1}}^{*} \Psi_{m_{2}, l_{2}} \Psi_{m_{3}, l_{3}}\right\rangle=\frac{\sqrt{m_{1} m_{2} m_{3}}}{\sqrt{2 \pi^{3}}} \int d w_{1} d w_{2} d w_{3} e^{i\left(-l_{1} w_{1}+l_{2} w_{2}-l_{3} w_{3}\right)} e^{\frac{i}{2} \theta m_{2} m_{3} \sinh \left(w_{2}-w_{3}\right)} \\
& \times \delta\left(-m_{1} e^{w_{1}}+m_{2} e^{w_{2}}+m_{3} e^{w_{3}}\right) \delta\left(-m_{1} e^{-w_{1}}+m_{2} e^{-w_{2}}+m_{3} e^{-w_{3}}\right) \delta^{(p-1)}\left(-\vec{k}_{1}+\vec{k}_{2}+\vec{k}_{3}\right) .
\end{aligned}
$$

The phase shift has been simplified by using the delta functions as in the two point function. The integration w.r.t $w_{1}$ leads to

$$
\begin{aligned}
& \frac{\sqrt{m_{1} m_{2} m_{3}}}{\sqrt{2 \pi^{3}}} \int d w_{2} d w_{3} e^{i\left(l_{2} w_{2}+l_{3} w_{3}\right)} \frac{\left(m_{2} e^{-w_{2}}+m_{3} e^{-w_{3}}\right)^{i l_{1}-1}}{m_{1}^{i l_{1}}} e^{\frac{i}{2} \theta m_{2} m_{3} \sinh \left(w_{2}-w_{3}\right)} \\
& \times \delta\left(-\frac{m_{1}^{2}}{m_{2} e^{-w_{2}}+m_{3} e^{-w_{3}}}+m_{2} e^{w_{2}}+m_{3} e^{w_{3}}\right) \delta^{(p-1)}\left(-\vec{k}_{1}+\vec{k}_{2}+\vec{k}_{3}\right) .
\end{aligned}
$$

Rewriting $w_{ \pm}=\frac{1}{2}\left(w_{2} \pm w_{3}\right)$ and integrating $w_{+}$out, we find

$$
\begin{aligned}
& 2 \sqrt{\frac{2 m_{1} m_{2} m_{3}}{\pi}} \delta_{l_{1}, l_{2}+l_{3}} \int d w_{-} e^{i\left(l_{2}-l_{3}\right) w_{-}} \frac{\left(m_{2} e^{-w_{-}}+m_{3} e^{w_{-}}\right)^{i l_{1}-1}}{m_{1}^{i l_{1}}} e^{\frac{i}{2} \theta m_{2} m_{3} \sinh 2 w_{-}} \\
& \times \delta\left(\frac{-m_{1}^{2}+m_{2}^{2}+m_{3}^{3}+2 m_{2} m_{3} \cosh 2 w_{-}}{m_{2} e^{-w_{-}}+m_{3} e^{w_{-}}}\right) \delta^{(p-1)}\left(-\vec{k}_{1}+\vec{k}_{2}+\vec{k}_{3}\right) .
\end{aligned}
$$


Assuming $m_{1}>m_{2}+m_{3}$, we finally obtain by integrating $w_{-}$out as

$$
\sqrt{\frac{2 m_{1} m_{2} m_{3}}{\pi}} \frac{\delta_{l_{1}, l_{2}+l_{3}}\left(e^{i \xi_{+}}+e^{i \xi_{-}}\right)}{M},
$$

where

$$
\begin{aligned}
& M=\sqrt{m_{1}^{2}-\left(m_{2}+m_{3}\right)^{2}} \sqrt{m_{1}^{2}-\left(m_{2}-m_{3}\right)^{2}} \\
& e^{i \xi_{ \pm}}=e^{ \pm \frac{i}{4} \theta M} e^{ \pm i\left(l_{2}-l_{3}\right) w_{0}}\left(\frac{m_{2} e^{\mp w_{0}}+m_{3} e^{ \pm w_{0}}}{m_{1}}\right)^{i l_{1}} \\
& \sinh w_{0}=\sqrt{\frac{m_{1}^{2}-\left(m_{2}+m_{3}\right)^{2}}{4 m_{2} m_{3}}}
\end{aligned}
$$

From the above result, we can see that the introduction of gauge flux affects the amplitude in a non-trivial way, though the amplitude is finite even without flux. Note that the shift due to the non-commutativity is very small for the case when the mass difference is very small $\left(m_{1} \sim m_{2}+m_{2}\right)$.

\subsection{Four point function}

We can compute four point function in the same manner as before. Both for closed and open strings, the $2 \rightarrow 2$ scattering amplitudes are written in the form of ${ }^{24}$

$$
\begin{aligned}
& \left\langle\Psi_{m_{3}, l_{3}}^{*} \Psi_{m_{4}, l_{4}}^{*} \Psi_{m_{1}, l_{1}} \Psi_{m_{2}, l_{2}}\right\rangle=\frac{\sqrt{m_{1} m_{2} m_{3} m_{4}}}{\pi} \delta^{(p-1)}\left(\sum_{i=1}^{4} \epsilon_{i} \vec{k}_{i}\right) \delta\left(\sum_{i=1}^{4} \epsilon_{i} l_{i}\right) \\
& \quad \times \int d v_{2} d v_{3} d v_{4} A(s, t, u) \delta\left(m_{1}+\prod_{i=2}^{4} \epsilon_{i} m_{i} v_{i}\right) \delta\left(m_{1}+\prod_{i=2}^{4} \epsilon_{i} m_{i} / v_{i}\right) \prod_{i=2}^{4} v_{i}^{i \epsilon_{i} l_{i}-1}
\end{aligned}
$$

where $\epsilon_{1}=\epsilon_{2}=-\epsilon_{3}=-\epsilon_{4}=1$. We have used $v_{i}=e^{w_{i}-w_{1}}(i=2,3,4)$ and the Mandelstam variables

$$
\begin{aligned}
& s=-\left(k_{1}+k_{2}\right)^{2}=2 m_{T}^{2}+m_{1} m_{2}\left(v_{2}+\frac{1}{v_{2}}\right)-2 \vec{k}_{1} \cdot \vec{k}_{2}, \\
& t=-\left(k_{1}-k_{3}\right)^{2}=2 m_{T}^{2}-m_{1} m_{3}\left(v_{3}+\frac{1}{v_{3}}\right)-2 \vec{k}_{1} \cdot \vec{k}_{3}, \\
& u=-\left(k_{1}-k_{4}\right)^{2}=2 m_{T}^{2}-m_{1} m_{4}\left(v_{4}+\frac{1}{v_{4}}\right)-2 \vec{k}_{1} \cdot \vec{k}_{4} .
\end{aligned}
$$

The tachyon mass is $m_{T}^{2}=-4 / \alpha^{\prime}$ for closed strings and $m_{T}^{2}=-1 / \alpha^{\prime}$ for open strings. Note that there is a non-trivial relation as $s+t+u=4 m_{T}^{2}$. For four point function, we

\footnotetext{
${ }^{24}$ For closed strings we have to set $p=25$.
} 
cannot fix the position $z$ of one of the four vertex operators, and $A(s, t, u)$ represents the contribution coming from the integration of $z$. The amplitude is called as Virasoro-Shapiro amplitude for closed strings and Veneziano amplitude for open strings.

In the closed string case, Virasoro-Shapiro amplitude is defined as

$$
\begin{aligned}
A_{V S}(s, t, u) & =\int d^{2} z|z|^{-\alpha^{\prime} u / 2-4}|1-z|^{-\alpha^{\prime} t / 2-4} \\
& =2 \pi \frac{\Gamma\left(-1-\frac{\alpha^{\prime} s}{4}\right) \Gamma\left(-1-\frac{\alpha^{\prime} t}{4}\right) \Gamma\left(-1-\frac{\alpha^{\prime} u}{4}\right)}{\Gamma\left(2+\frac{\alpha^{\prime} s}{4}\right) \Gamma\left(2+\frac{\alpha^{\prime} t}{4}\right) \Gamma\left(2+\frac{\alpha^{\prime} u}{4}\right)} .
\end{aligned}
$$

Later we will use its high energy behavior. If we take $s, t, u$ very large, then the amplitude is exponentially damping. On the other hand, in the Regge limit, where $s \rightarrow \infty$ and $t$ is fixed, the amplitude behaves as

$$
A_{V S} \sim s^{2+\frac{\alpha^{\prime} t}{2}} \frac{\Gamma\left(-1-\frac{\alpha^{\prime} t}{4}\right)}{\Gamma\left(2+\frac{\alpha^{\prime} t}{4}\right)} .
$$

The poles of the gamma function at $\alpha^{\prime} t=-4,0,4, \cdots$ correspond to the on-shell poles of exchanged states, and the powers of $s$ are the spins of the states. For example, the tachyon $m^{2}=-4 / \alpha^{\prime}$ has spin 0 , the graviton $m^{2}=0$ has spin 2 , and so on.

Since we introduce non-trivial flux on the D-brane in open string case, the Veneziano amplitude is modified due to the non-commutativity. Taking care of the ordering of the four vertex operators and making use of the momentum conservation, we can write the amplitude as $^{25}$

$$
\begin{aligned}
A_{V}(s, t, u) & =\left(e^{i\left(-\chi_{42}-\chi_{43}+\chi_{23}\right)}+e^{i\left(-\chi_{42}-\chi_{43}+\chi_{23}\right)}\right) I(s, t) \\
& +\left(e^{i\left(-\chi_{42}+\chi_{43}-\chi_{23}\right)}+e^{i\left(+\chi_{42}-\chi_{43}+\chi_{23}\right)}\right) I(t, u) \\
& +\left(e^{i\left(-\chi_{24}+\chi_{34}+\chi_{23}\right)}+e^{i\left(+\chi_{42}-\chi_{43}-\chi_{23}\right)}\right) I(s, u),
\end{aligned}
$$

where

$$
I(x, y)=\int_{0}^{1} d y y^{-\alpha^{\prime} x-2}(1-y)^{-\alpha^{\prime} t-2}=\frac{\Gamma\left(-\alpha^{\prime} x-1\right) \Gamma\left(-\alpha^{\prime} y-1\right)}{\Gamma\left(-\alpha^{\prime} x-\alpha^{\prime} y-2\right)}
$$

and

$$
\chi_{i j}=\frac{1}{4} m_{i} m_{j}\left(\frac{v_{i}}{v_{j}}-\frac{v_{j}}{v_{i}}\right) .
$$

The high energy behavior of Veneziano amplitude is very similar to the Virasoro-Shapiro amplitude. Again an interesting behavior is shown in the Regge limit as

$$
I(s, t) \sim I(t, u) \sim s^{1+\alpha^{\prime} t} \Gamma\left(-1-\alpha^{\prime} t\right)
$$

\footnotetext{
${ }^{25}$ Using the cyclic property of disk amplitude, we set $\tau_{1}$ less than the other $\tau_{i}$. Then, the phase factor involving $m_{1}$ and $w_{1}$ becomes trivial due to the momentum conservation. The other part of phase factor depends on the ordering of $\tau_{i}$ because of the epsilon factor in (5.3).
} 
with exponentially damping $I(s, u)$. The poles of exchanged states are at $\alpha^{\prime} t=-1,0,1, \cdots$. The powers of $s$ are spins of the states, which are 0 for the tachyon $\left(m^{2}=-1 / \alpha^{\prime}\right), 1$ for gauge field $\left(m^{2}=0\right)$, and so forth.

Now that we know the properties of the amplitude $A(s, t, u)$, let us perform the integration of $v_{i}$ in (5.11). Using the delta functions, the four point function can be written as

$$
\frac{\sqrt{m_{1} m_{2} m_{3} m_{4}}}{\pi} \delta^{(p-1)}\left(\sum_{i=1}^{4} \epsilon_{i} \vec{k}_{i}\right) \delta\left(\sum_{i=1}^{4} \epsilon_{i} l_{i}\right) \int d v_{4} A(s, t, u) \frac{v_{2}^{i l_{2}+1} v_{3}^{i l_{3}+1} v_{4}^{i l_{4}-1}}{m_{2} m_{3}\left|v_{2}^{2}-v_{3}^{2}\right|}
$$

where $v_{2}$ and $v_{3}$ are replaced by

$$
v_{2}=\frac{\alpha \beta+m_{2}^{2}-m_{3}^{2} \mp \sqrt{\Xi}}{2 m_{2} \beta}, \quad v_{3}=-\frac{\alpha \beta+m_{3}^{2}-m_{2}^{2} \pm \sqrt{\Xi}}{2 m_{3} \beta}
$$

with

$$
\alpha=-m_{1}+m_{4} v_{4}, \quad \beta=-m_{1}+\frac{m_{4}}{v_{4}}, \quad \Xi=\left(m_{2}^{2}-m_{3}^{2}\right)^{2}-2 \alpha \beta\left(m_{2}^{2}+m_{3}^{2}\right)+\alpha^{2} \beta^{2} .
$$

The integral w.r.t. $v_{4}$ may diverge at the high energy region $v_{4} \rightarrow \infty$. In $v_{4} \rightarrow \infty$ limit, we find

$$
v_{2} \sim \frac{m_{4} v_{4}}{m_{2}}, \quad v_{3} \sim \frac{m_{3}}{m_{1}}, \quad t \sim-\left(\vec{k}_{1}-\vec{k}_{3}\right)^{2}, \quad s \sim m_{1} m_{4} v_{4},
$$

therefore we can see that the limit corresponds to the Regge limit.

First, we examine the closed string case, which was already done in $\left[18 .{ }^{26}\right.$ Using the Regge limit of Virasoro-Shapiro amplitude, the four point function becomes

$$
\frac{\Gamma\left(-1+\frac{\alpha^{\prime}\left(\vec{k}_{1}-\vec{k}_{3}\right)^{2}}{4}\right)}{\Gamma\left(2-\frac{\alpha^{\prime}\left(\vec{k}_{1}-\vec{k}_{3}\right)^{2}}{4}\right)} \int_{v_{m}}^{\infty} d v_{4} v_{4}^{-\frac{\alpha^{\prime}}{2}\left(\vec{k}_{1}-\vec{k}_{3}\right)^{2}+i\left(l_{2}-l_{4}\right)},
$$

where we take a cut off $v_{m} \gg 1$. The integral diverges for $\left(\vec{k}_{1}-\vec{k}_{3}\right)^{2} \leq \frac{2}{\alpha^{\prime}}$ and $l_{2}-l_{4}=0$, where graviton is exchanged. From this fact, it was claimed in [18 that there is large back reaction due to the graviton exchange near the big crunch/big bang singularity. Note that there are other types of sources of divergences coming from the poles of the gamma function. Since they are on-shell poles of exchanged states, the divergence originates from the IR effects, where this kind of divergence is usually regularized by the $i \epsilon$-prescription. The UV divergence at $v_{4} \rightarrow \infty$ also comes from IR region $\left(\vec{k}_{1}-\vec{k}_{3}\right)^{2} \leq \frac{2}{\alpha^{\prime}}$, and hence this phenomenon may be regarded as an example of IR/UV mixing [6, 9, 18.

For our open string case, we can expect that the divergence becomes milder since the spin in the contribution $s^{\text {spin }} \sim v_{4}^{\text {spin }}$ is changed from spin $=2$ into spin $=1$, roughly

\footnotetext{
${ }^{26}$ See [6, 9] for null brane case.
} 
speaking. In fact, for open strings on D-brane without flux, the four point function behaves as

$$
\Gamma\left(-1+\alpha^{\prime}\left(\vec{k}_{1}-\vec{k}_{3}\right)^{2}\right) \int_{v_{m}}^{\infty} d v_{4} v_{4}^{-1-\alpha^{\prime}\left(\vec{k}_{1}-\vec{k}_{3}\right)^{2}+i\left(l_{2}-l_{4}\right)},
$$

therefore the integral diverges only when $\left(\vec{k}_{1}-\vec{k}_{3}\right)^{2}=0$ and $l_{2}-l_{4}=0$. This is actually much better than in the closed string case, because the singularity may be removed by $i \epsilon$-prescription just like for the on-shell divergences, which cannot be used to regularize the divergence in the closed string amplitude. ${ }^{27}$

Finally, let us see whether the non-commutativity resolves the singularity. Because the phase factor in (5.15) behaves as

$$
\chi_{42} \sim \mathcal{O}(1), \quad \chi_{43} \sim \chi_{23} \sim \frac{1}{4} m_{1} m_{2} v_{4},
$$

only a constant factor is added to (5.24). Therefore, we conclude that the non-commutativity does not resolve the singularity in this case. This might be consistent with the fact that only non-planer loop amplitudes would be regularized by the introduction of noncommutativity (see, e.g., [71]).

\section{Strings and D-branes in Grant space}

As discussed in [9, 10, 11, the singular behavior of closed strings associated to the big crunch/big bang is largely improved by adding non-trivial directions to the parabolic orbifold. In our case, we introduce one more dimension $y$ and require the identification

$$
x^{ \pm} \sim e^{ \pm 2 \pi \gamma} x^{ \pm}, \quad y \sim y+2 \pi R .
$$

This space is called as Grant space [46, where the singular point at $x^{+}=x^{-}=0$ disappears due to the translation along $y$ direction. We should remark that the space still includes closed time-like curves. In this section, we examine how the resolution of the singularity affects the (imaginary part of) annulus amplitudes for open strings and open string scattering amplitudes.

\subsection{Torus amplitude}

The spectrum of closed strings in Grant space is almost the same as the product of those for Misner space and for a free boson $Y$. Only the difference is that the conjugate

\footnotetext{
${ }^{27}$ Since the divergence arises at $t=0$, the divergence may be regarded as IR/UV mixing as in the closed string case (see also [30). Moreover, we can show that the contribution of the divergence comes from the region near the big crunch/big bang singularity following [18. However, we cannot identify the divergence as the signal of the large back reaction since the open string tachyon does not couple to dilaton or graviton in the tree level. It would be interesting to examine the open string one loop effects, which may be interpreted as closed string exchanges in string theory context.
} 
momentum for $y$ direction is restricted as $P_{y}=\frac{1}{R}(n-\gamma \hat{J})$, where $p_{y}=\frac{1}{2}\left(P_{y}+\frac{w R}{\alpha^{\prime}}\right)$ and $\tilde{p}_{y}=\frac{1}{2}\left(P_{y}-\frac{w R}{\alpha^{\prime}}\right)$ for left and right-movers, respectively. Therefore, we can compute torus amplitude in the way similar to the calculation in Misner space. Since the amplitude was already obtained in [3] in the oscillator formalism, we re-derive it in the path integral formalism.

As before, we concentrate on the twisted sector. In the $(w, k)$-twisted sector, the boundary condition for $Y$ is assigned as

$$
Y\left(\sigma_{1}+2 \pi, \sigma_{2}\right)=2 \pi R w+Y\left(\sigma_{1}, \sigma_{2}\right), \quad Y\left(\sigma_{1}, \sigma_{2}+2 \pi\right)=2 \pi R k+Y\left(\sigma_{1}, \sigma_{2}\right)
$$

along with (2.12), and the mode expansion is given by

$$
Y\left(\sigma_{1}, \sigma_{2}\right)=y_{0}+R\left(w \sigma_{1}+k \sigma_{2}\right)+\sum_{\{m, n\} \neq\{0,0\}} a_{m, n} e^{i\left(m \sigma_{1}+n \sigma_{2}\right)} .
$$

While evaluating the path integral, a subtlety arises from the overall normalization, which is fixed by following [72, 47]. Namely, we separate constant and orthogonal parts as $Y\left(\sigma_{i}\right)=\tilde{Y}+Y^{\prime}\left(\sigma_{i}\right)$ and normalize as

$$
\int \mathcal{D} \delta Y e^{-\frac{1}{4 \pi \alpha} \int d^{2} \sigma \sqrt{g}(\delta Y)^{2}}=1
$$

Therefore, we have

$$
\int \mathcal{D} \delta Y^{\prime} e^{-\frac{1}{4 \pi \alpha} \int d^{2} \sigma \sqrt{g}\left(\delta Y^{\prime}\right)^{2}}=\left(\int d y e^{-\frac{1}{4 \pi \alpha} \int d^{2} \sigma \sqrt{g} y^{2}}\right)^{-1}=\left(\frac{\pi}{\frac{1}{4 \pi \alpha^{\prime}} \int d^{2} \sigma \sqrt{g}}\right)^{-1 / 2}=\sqrt{\frac{\tau_{2}}{\alpha^{\prime}}} .
$$

Using this normalization, the partition function for the $Y$ part is written as

$$
\mathcal{T}_{w, k}^{Y}(\tau)=\sqrt{\frac{\tau_{2}}{\alpha^{\prime}}} \operatorname{Det}^{\prime-1 / 2}(-\Delta) e^{-\frac{\pi R^{2}}{\alpha^{\prime} \tau_{2}}|k-\tau w|^{2}},
$$

where the contribution from non-zero modes is computed as

$$
\operatorname{Det}^{\prime}(-\Delta)=\prod_{\{m, n\} \neq\{0,0\}} \frac{1}{\tau_{2}^{2}}|n-\tau m|^{2}=4 \pi^{2} \tau_{2}^{2}|\eta(\tau)|^{4}
$$

Summing up all contributions, we obtain

$$
\mathcal{T}_{w, k}(\tau)=\frac{1}{\sqrt{4 \pi^{2} \alpha^{\prime} \tau_{2}}} \frac{e^{-\frac{\pi R^{2}}{\alpha^{\prime} \tau_{2}}|k-\tau w|^{2}-2 \pi \tau_{2} \gamma^{2} w^{2}}}{\left|\vartheta_{1}(i \gamma(k-\tau w) \mid \tau)\right|^{2}},
$$

which reproduces the result in [3]. ${ }^{28}$ Adding extra 23 free bosons and ghost parts, we have the total amplitude

$$
\mathcal{T}=\sum_{k, w} \int_{\mathcal{F}} \frac{d^{2} \tau}{4 \pi^{2} \alpha^{\prime} \tau_{2}^{2}} \frac{e^{-\frac{\pi R^{2}}{\alpha^{\prime} \tau_{2}}|w-\tau k|^{2}-2 \pi \tau_{2} \gamma^{2} w^{2}}}{\left(4 \pi^{2} \alpha^{\prime} \tau_{2}\right)^{11}\left|\vartheta_{1}(i \gamma(w \tau+k) \mid \tau) \eta(\tau)^{21}\right|^{2}}
$$

Notice that the amplitude receives suppression for large $|w|$ and/or $|k|$ from the new factor.

\footnotetext{
${ }^{28}$ Notice that we have neglected total volume factor, i.e., $2 \pi R$ in this case.
} 


\subsection{Annulus amplitudes}

We have investigated D-branes in Misner space, and found the divergent rates of open string pair creation and emission of winding strings. Since singular behaviors of closed strings are improved by introducing extra non-trivial direction in null brane case 9, 10, 11, we expect that the string emission rates are suppressed also in our case. From this motivation, we will compute the string emission rates from various D-branes in Grant space. For simplicity, we assign Neumann or Dirichlet boundary condition to $Y$, thus we have D0-brane, D1-brane and D2-brane. Notice that there are two types of D1-branes which are localized in the spatial direction of Misner space and in the extra direction $y$.

We first examine the $\mathrm{D} p$-brane localized in the spatial directions of Grant space (D0brane in Grant space part). For the Misner space case, localized D $p$-brane has been constructed by summing over all image branes in the covering space, and the same approach should be taken also for the Grant space case. Let us assume that the original brane is located at $y=y_{0}$ in the covering space, then the $k$-th image brane is at $y=y_{0}+2 \pi R k$. The open string stretched between $k$-th and $k^{\prime}$-th image branes has the boundary conditions

$$
\partial_{\tau} Y(\tau, 0)=\partial_{\tau} Y(\tau, \pi)=0, \quad Y(\tau, 0)=y_{0}+2 \pi R k, \quad Y(\tau, \pi)=y_{0}+2 \pi R k^{\prime}
$$

The mode expansion is

$$
Y=y_{0}+2 \pi R k+2 R\left(k^{\prime}-k\right) \sigma+i \sqrt{2 \alpha^{\prime}} \sum_{n \in \mathbb{Z}, n \neq 0} \frac{\alpha_{n}^{y}}{n} e^{i n \tau} \sin n \sigma,
$$

and the Virasoro generator is

$$
L_{0}=\frac{\left|k-k^{\prime}\right|^{2} R^{2}}{\alpha^{\prime}}+\sum_{n>0} \alpha_{-n}^{y} \alpha_{n}^{y} .
$$

Since the contribution to the annulus amplitude is computed as

$$
\operatorname{Tr} e^{-2 \pi t\left(L_{0}-\frac{1}{24}\right)}=\frac{e^{-\frac{2 \pi t\left|k-k^{\prime}\right|^{2} R^{2}}{\alpha^{\prime}}}}{\eta(i t)}
$$

we obtain $\left(\nu=2 \gamma\left(k-k^{\prime}\right)\right)$

$$
\mathcal{A}=\sum_{k, k^{\prime}} \int_{0}^{\infty} \frac{d t}{t} \frac{e^{-\pi t \nu^{2}-\frac{2 \pi t\left|k-k^{\prime}\right|^{2} R^{2}}{\alpha^{\prime}}}}{\left(8 \pi^{2} \alpha^{\prime} t\right)^{\frac{p}{2}} \vartheta_{1}(t \mid \nu \| i t) \eta(i t)^{21}} .
$$

Here we have added 23 extra boson contribution. The imaginary part arises from the poles of integration by $t$ as

$$
-2 \operatorname{Im} \mathcal{A}=\sum_{k, k^{\prime}} \sum_{n=1}^{\infty} \frac{(-1)^{n+1}}{n\left(8 \pi^{2} \alpha^{\prime}\right)^{\frac{p}{2}}}\left(\frac{|\nu|}{n}\right)^{\frac{p}{2}} \sum_{\text {states }} e^{-\frac{2 \pi n}{|\nu|}\left(\frac{R^{2}\left|k-k^{\prime}\right|^{2}}{\alpha^{\prime}}+N-1\right)-\pi n|\nu|} .
$$


This value is suppressed for large $\left|k-k^{\prime}\right|$ from the new factor, even though the rate is still divergent due the the Hagedorn density $\rho_{N} \sim e^{2 \pi N}$ for $n /|\nu|<1$. This suppression factor can be understood as follows. The pair created open strings are stretched between image branes also in $y$ direction, thus the open strings acquire extra mass and hence the pair creation rate is suppressed.

Next we move to D1-brane extended to $y$ direction in Grant space. In this case, we assign Neumann boundary condition to $Y$ field, and this gives no changes from computation on the $\mathrm{D} p$-brane in Misner space case with $p \geq 1$. Therefore, the pair creation rate of open strings is not altered from (3.42), and hence there is no effect of introducing extra non-trivial direction.

In Grant space, there is another type of D1-brane, which wraps the whole $x^{ \pm}$part but is localized in the $y$ direction. Since the brane does not wrap the whole Grant space, only untwisted closed strings couple to the D-brane. Thus the $X^{ \pm}$part is the same as the flat space case, and $Y$ part is the same as D0-brane in Grant space, namely, the all image branes at $y=y_{0}+2 \pi k R$ should be taken into account. The annulus amplitude for the corresponding $\mathrm{D} p$-brane is then given as

$$
\mathcal{A}=\sum_{k, k^{\prime}} \int_{0}^{\infty} \frac{d t}{t} \frac{e^{-\frac{2 \pi t\left|k-k^{\prime}\right|^{2} R^{2}}{\alpha^{\prime}}}}{\left(8 \pi^{2} \alpha^{\prime} t\right)^{\frac{p}{2}} \eta(i t)^{24}}
$$

and it does not include imaginary part. Therefore, we can say that the divergence of winding string emission is completely removed. This is because only winding closed strings would be emitted from the D-brane wrapping $x^{ \pm}$part as seen in section 3 but the winding strings do not couple to this type of D-brane.

Finally, let us analyze D2-brane wrapping the whole Grant space part. In the path integral formulation, we have boundary conditions for $Y$

$$
Y\left(\sigma_{1}, \sigma_{2}+2 \pi\right)=2 \pi R k+Y\left(\sigma_{1}, \sigma_{2}\right), \quad \partial_{\sigma_{1}} Y\left(\sigma_{1}=0, \pi, \sigma_{2}\right)=0
$$

along with (3.55) and (3.56), whose solution is

$$
Y\left(\sigma_{1}, \sigma_{2}\right)=y_{0}+R k \sigma_{2}+\sum_{\{m, n\} \neq\{0,0\}, m \geq 0} a_{m, n}^{y} \cos m \sigma_{1} e^{i n \sigma_{2}} .
$$

Therefore we multiply the Misner part by

$$
\mathcal{A}_{k}^{Y}=\frac{e^{-\frac{\pi R^{2} k^{2}}{2 \alpha^{\prime} t}}}{\left(8 \pi^{2} \alpha^{\prime} t\right)^{\frac{1}{2}} \eta(i t)}=\frac{e^{-\frac{\pi s R^{2} k^{2}}{2 \alpha^{\prime}}}}{\left(8 \pi^{2} \alpha^{\prime}\right)^{\frac{1}{2}} \eta(i s)} .
$$

In the oscillator formalism, we should take the twist operator into account. Since the $Y$ satisfies the Neumann boundary condition, the contribution is computed as

$$
\begin{aligned}
\mathcal{A}_{k}^{Y} & =\operatorname{Tr}\left(e^{2 \pi i R p_{y}}\right)^{k} e^{-2 \pi t\left(L_{0}-\frac{1}{24}\right)} \\
& =\int \frac{d p_{y}}{2 \pi} \frac{e^{2 \pi i k R p_{y}-2 \pi t\left(\alpha^{\prime} p_{y}{ }^{2}-\frac{1}{24}\right)}}{\prod_{n>0}\left(1-e^{-2 \pi t n}\right)}=\int \frac{d p_{y}}{2 \pi} \frac{e^{-2 \pi t \alpha^{\prime}\left(p_{y}-\frac{i R k}{2 t \alpha^{\prime}}\right)^{2}-\frac{\pi R^{2} k^{2}}{2 \alpha^{\prime} t}+\frac{\pi t}{12}}}{\prod_{n>0}\left(1-e^{-2 \pi t n}\right)},
\end{aligned}
$$


which reproduces the path integral result. Incorporating this part, we obtain the total cylinder amplitude as $(\nu=\gamma k)$

$$
\mathcal{A}=\sum_{k \neq 0} \int_{0}^{\infty} d s \frac{e^{-\pi s \nu^{2}-\frac{\pi s R^{2} k^{2}}{2 \alpha^{\prime}}}}{2 \sinh (\pi|\nu|)\left(8 \pi^{2} \alpha^{\prime}\right)^{\frac{p-1}{2}} s^{\frac{25-p}{2}} \vartheta_{1}(|\nu| s \mid i s) \eta(i s)^{21}}
$$

This leads to imaginary part from the poles of integration by $s$ as

$$
-2 \operatorname{Im} \mathcal{A}=\sum_{w \neq 0} \sum_{n=1}^{\infty} \frac{(-1)^{n+1}}{2|\nu| \sinh (\pi|\nu|)\left(8 \pi^{2} \alpha^{\prime}\right)^{\frac{p-1}{2}}}\left(\frac{|\nu|}{n}\right)^{\frac{25-p}{2}} \sum_{\text {states }} e^{-\frac{2 \pi n}{|\nu|}\left(\frac{R^{2} k^{2}}{4 \alpha^{\prime}}+N-1\right)-\pi n|\nu|} .
$$

Therefore, as in the D0-brane case, there is suppression from the new factor for large $|k|$, even though the emission rate of winding strings is still divergent for $n /|\nu|<1$ due to the Hagedorn behavior $\rho_{N} \sim e^{2 \pi N}$. This suppression comes from the fact that the closed strings wrap the $y$ direction as well in the twisted sectors, which gives extra mass to the emitted winding strings.

\subsection{Four point function}

As seen in section 5, the $2 \rightarrow 2$ scattering amplitude for open strings diverges due to the big crunch/big bang singularity. Therefore, it is important to examine if the introduction of extra direction resolves this singular behavior. We use the $\mathrm{D} p$-brane without flux wrapping the whole Grant space. The wave function is defined as

$$
\Psi_{m, l, n, \vec{p}}=\sqrt{\frac{m}{2 \pi R}} \int d w e^{\frac{i m}{\sqrt{2}}\left(X^{-} e^{-w}+X^{+} e^{w}\right)+i w l+i p_{y} y+i \vec{p} \cdot \vec{X}}
$$

where $p_{y}=(n-\gamma l) / R$. A crucial difference from the wave function for the Misner space case (5.1) is that now $l$ takes a real value, and hence the wave function behaves badly around $X^{+} X^{-} \sim 0[18,[21] \text {. Therefore, it is more plausible to use a wave packet [21] }]^{29}$

$$
\Psi_{m, f(l), n, \vec{p}}=\int d l f(l) \Psi_{m, l, n, \vec{p}}
$$

with a smooth function of rapid decrease $f(l)$. Then, the four point function of the wave packets is computed by ${ }^{30}$

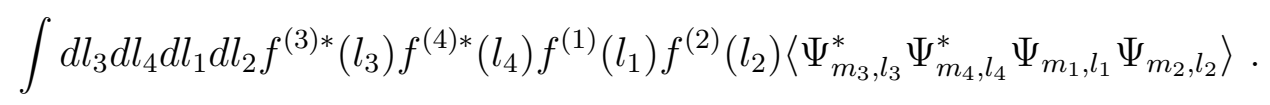

Following the appendix of [21], the four point function can be evaluated as $\left(v_{4}=e^{\sigma}\right)$

$$
\left.\int d \sigma e^{-\sigma \alpha^{\prime}\left|\vec{p}_{1}-\vec{p}_{3}\right|^{2}}\left[\left(\tilde{f}^{(1)} \tilde{f}^{(3)}(x)\right) *\left(\tilde{f}^{(2) *} \tilde{f}^{(4) *}(x)\right) *\left(\frac{1}{\sqrt{\sigma}} e^{-\frac{x^{2} R^{2}}{4 \sigma \alpha^{\prime} \gamma^{2}}}\right) *\left(e^{-\frac{|x|\left|\vec{p}_{1}-\vec{p}_{3}\right| R}{\gamma}}\right)\right]\right|_{x=\sigma}
$$

\footnotetext{
${ }^{29}$ See [9] for null brane case.

${ }^{30}$ We assume $f^{*}(l)=f(-l)$ such that the formula would be simpler.
} 
in the large $v_{4}$ region. We denote the Fourier transform of $f(l)$ as $\tilde{f}(x)$ and the convolution product as $*$. We have also set $n_{1}=n_{3}$ in order to see the singular behavior. Assuming that $f^{(i)}(l)$ decay faster enough, the four point function is approximated as

$$
\int d \sigma e^{-\sigma\left(\alpha^{\prime}\left|\vec{p}_{1}-\vec{p}_{3}\right|^{2}+\frac{R^{2}}{4 \alpha^{\prime} \gamma^{2}}+\frac{\left|\vec{p}_{1}-\vec{p}_{3}\right| R}{\gamma}\right)} .
$$

We can see that the integral converges even around $\left|\vec{p}_{1}-\vec{p}_{3}\right|=0$. Therefore, we conclude that the divergence is removed by introduction of extra non-trivial direction just like the null brane case [9, 10, 11]. In fact, this is contrary to the closed string case where the divergence on the four point function cannot be completely removed in the Grant space 21]. We should remark that we can reproduce the previous result by taking the limit $R \rightarrow 0$.

\section{Conclusion}

In this paper we have studied various aspects of D-branes and open strings on them in a space-time that contains big crunch/big bang singularity. As a simple setup, we have chosen a Lorentzian orbifold $\mathbb{R}^{1,1} / \Gamma$ with a discrete boost $\Gamma$, which is known as Misner (Milne) space [17]. The construction is very simple thanks to the orbifold technique, but the structure is complex enough; The universe includes cosmological regions with big crunch and big bang singularities and whisker regions with closed time-like curves (CTCs). First, we have examined the spectrum of closed strings, mainly, in the twisted sectors of the orbifold model, where the winding strings can be of macroscopic size [19, 20, 21, 22. We have also computed the one-loop amplitude [4, and shown the no-ghost theorem. In particular, we have noticed that only transverse excitations contribute to the one-loop amplitude.

In the Misner space, there are D0-brane and D1-brane as in fig. 1. The D0-brane starts from the past infinity, meets the big crunch singularity, passes through the regions with CTC, and goes to the future infinity. The D1-brane wraps the whole universe, and can include gauge flux, which induces time-dependent non-commutativity in the effective theory. We have computed the partition functions in terms of both one-loop amplitudes for open strings and overlaps between boundary states. Interestingly, we have observed the imaginary parts of the amplitudes for both the D0-brane and the D1-brane, which have been interpreted as follows. In order to construct the D0-brane in the orbifold model, we have to sum up the image branes generated by the discrete boost in the covering space. The imaginary part of the annulus amplitude corresponds to the absorption rate between image branes, and this can be also interpreted as the pair creation rate of open strings 56. For the D1-brane, the imaginary part is understood as the emission of twisted closed

strings. Since the emitted winding strings are localized in whisker regions, the emission may be related to the chronology protection conjecture [24]. We have extended this 
analysis into superstring case to show that the cause of the D-brane instabilities is not related to the tachyonic state, which would be projected out in the superstring settings.

It was already known [18] that the $2 \rightarrow 2$ scattering amplitude for closed strings in Misner space is divergent due to the graviton exchanges near the big crunch/big bang singularity. We have examined $2 \rightarrow 2$ scattering for open strings on D-branes wrapping the Misner space part, and found that the divergent structure is largely improved since gauge field exchanges instead of graviton. On the D-brane wrapping the Misner space, we can include non-trivial gauge flux, and the flux induces time-dependent non-commutativity. However, we have shown that the non-commutativity does not resolve the divergence associated big crunch/big bang singularity in the 4 point function.

Including an extra direction and taking orbifold by combining boost and translation, we obtain a smooth manifold without big crunch/big bang singularity, which is called as Grant space [46. We have examined D-branes and open strings on them in Grant space. The rates of open string pair creation and emission of winding closed strings are mostly suppressed by introduction of extra non-trivial direction. We have shown that $2 \rightarrow 2$ open string scattering amplitude is also regularized by the effect.

To conclude, we have introduced D-branes as probes to investigate a big bang/big crunch universe, namely, the Misner space. Undoubtfully, a most important problem is to study the back reaction to this background. For example, we could not answer the question what will happen after the end of open string pair creation and emission of winding strings. A related question may be on the closed string tachyon condensation in the twisted sectors [24, 28. The twisted tachyon arises for bosonic string or superstrings with anti-periodic fermions. The tachyonic states are naively obtained by setting $\omega^{2}=\tilde{\omega}^{2}=-\mathcal{M}^{2}$ in (2.7), which may lead to the classical trajectories

$$
X^{ \pm}(\tau, \sigma)= \pm \frac{\sqrt{2 \alpha^{\prime}} \mathcal{M}}{\nu} e^{ \pm \nu \sigma} \sinh \nu \tau, \quad X^{ \pm}(\tau, \sigma)=\frac{\sqrt{2 \alpha^{\prime}} \mathcal{M}}{\nu} e^{ \pm \nu \sigma} \cosh \nu \tau
$$

for $\epsilon=\tilde{\epsilon}=1$ and $\epsilon=-\tilde{\epsilon}=1$, respectively. These states are not localized around the big crunch/big bang singularity, and are localized in the regions opposite to the massive case. For Euclidean orbifold case [67], the analysis of closed string tachyon becomes simpler, because the tachyon is localized at the fixed point. The analysis for Lorentzian orbifold case is rather difficult due to the non-locality of the twisted tachyon.

It is also interesting to apply this analysis into time-dependent non-commutative field theories and AdS/CFT correspondence, for example, along the line of [35, 37, 38. Since the effective theory on D-brane can be obtained by utilizing the orbifold methods, it might be possible to analyze the theory even with time-dependent non-commutativity. It is also worth to study open strings stretched between different D-branes. In this case, the structure of poles in moduli integral is changed, and hence the behavior of emissions of open and/or closed strings would be different. It might be important to study the scattering amplitude for open strings on D0-branes, which should be similar to scattering 
amplitudes for twisted closed strings [21].

\section{Acknowledgement}

We would like to thank Yu Nakayama, Makoto Natsuume, Soo-Jong Rey, Yuji Sugawara and Atsushi Yamaguchi for useful discussions. RRN and KLP wish to thank high energy theory group Institute of Physics, India, especially Alok Kumar, for the hospitality where a part of the work was done. The work of RRN and KLP was supported in part by INFN, by the MIUR-COFIN contract 2003-023852, by the EU contracts MRTN-CT2004-503369 and MRTN-CT-2004-512194, by the INTAS contract 03-51-6346, and by the NATO grant PST.CLG.978785.

\section{A Theta and eta functions}

We have used the definition of theta functions as

$$
\begin{aligned}
& \vartheta_{1}(\nu \mid \tau)=2 q^{\frac{1}{8}} \sin \pi \nu \prod_{m=1}^{\infty}\left(1-q^{m}\right)\left(1-z q^{m}\right)\left(1-z^{-1} q^{m}\right) \\
& \vartheta_{2}(\nu \mid \tau)=2 q^{\frac{1}{8}} \cos \pi \nu \prod_{m=1}^{\infty}\left(1-q^{m}\right)\left(1+z q^{m}\right)\left(1+z^{-1} q^{m}\right) \\
& \vartheta_{3}(\nu \mid \tau)=\prod_{m=1}^{\infty}\left(1-q^{m}\right)\left(1+z q^{m-\frac{1}{2}}\right)\left(1+z^{-1} q^{m-\frac{1}{2}}\right) \\
& \vartheta_{4}(\nu \mid \tau)=\prod_{m=1}^{\infty}\left(1-q^{m}\right)\left(1-z q^{m-\frac{1}{2}}\right)\left(1-z^{-1} q^{m-\frac{1}{2}}\right)
\end{aligned}
$$

where we have set $q=\exp (2 \pi i \tau)$ and $z=\exp (2 \pi i \nu)$. Their modular transformations are

$$
\begin{array}{ll}
\vartheta_{1}\left(\frac{\nu}{\tau} \mid-\frac{1}{\tau}\right)=-i(-i \tau)^{\frac{1}{2}} e^{\frac{\pi i \nu^{2}}{\tau}} \vartheta_{1}(\nu \mid \tau), & \vartheta_{2}\left(\frac{\nu}{\tau} \mid-\frac{1}{\tau}\right)=(-i \tau)^{\frac{1}{2}} e^{\frac{\pi i \nu^{2}}{\tau}} \vartheta_{4}(\nu \mid \tau), \\
\vartheta_{3}\left(\frac{\nu}{\tau} \mid-\frac{1}{\tau}\right)=(-i \tau)^{\frac{1}{2}} e^{\frac{\pi i \nu^{2}}{\tau}} \vartheta_{3}(\nu \mid \tau), & \vartheta_{4}\left(\frac{\nu}{\tau} \mid-\frac{1}{\tau}\right)=(-i \tau)^{\frac{1}{2}} e^{\frac{\pi i \nu^{2}}{\tau}} \vartheta_{2}(\nu \mid \tau) .
\end{array}
$$

We have also used Dedekind eta function defined as

$$
\eta(\tau)=q^{\frac{1}{12}} \prod_{m=1}^{\infty}\left(1-q^{m}\right), \quad \eta\left(-\frac{1}{\tau}\right)=(-i \tau)^{\frac{1}{2}} \eta(\tau) .
$$

\section{References}

[1] J. Khoury, B. A. Ovrut, N. Seiberg, P. J. Steinhardt and N. Turok, "From big crunch to big bang," Phys. Rev. D 65, 086007 (2002) arXiv:hep-th/0108187. 
[2] V. Balasubramanian, S. F. Hassan, E. Keski-Vakkuri and A. Naqvi, "A space-time orbifold: A toy model for a cosmological singularity," Phys. Rev. D 67, 026003 (2003) arXiv:hep-th/0202187.

[3] L. Cornalba and M. S. Costa, "A new cosmological scenario in string theory," Phys. Rev. D 66, 066001 (2002) arXiv:hep-th/0203031.

[4] N. A. Nekrasov, "Milne universe, tachyons, and quantum group," Surveys High Energ. Phys. 17, 115 (2002) arXiv:hep-th/0203112.

[5] J. Simon, "The geometry of null rotation identifications," JHEP 0206, 001 (2002) arXiv:hep-th/0203201.

[6] H. Liu, G. W. Moore and N. Seiberg, "Strings in a time-dependent orbifold," JHEP 0206, 045 (2002) arXiv:hep-th/0204168.

[7] L. Cornalba, M.S. Costa, and C. Kounnas, "A resolution of the cosmological singularity with orientifolds", Nucl. Phys. B637 378 (2002) arXiv: hep-th/0204261.

[8] A. Lawrence, "On the instability of 3D null singularities," JHEP 0211, 019 (2002) arXiv:hep-th/0205288.

[9] H. Liu, G. W. Moore and N. Seiberg, "Strings in time-dependent orbifolds," JHEP 0210, 031 (2002) arXiv:hep-th/0206182.

[10] M. Fabinger and J. McGreevy, "On smooth time-dependent orbifolds and null singularities," JHEP 0306, 042 (2003) arXiv:hep-th/0206196.

[11] G. T. Horowitz and J. Polchinski, "Instability of space-like and null orbifold singularities," Phys. Rev. D 66, 103512 (2002) arXiv:hep-th/0206228.

[12] C. R. Nappi and E. Witten, "A closed, expanding universe in string theory," Phys. Lett. B 293, 309 (1992) arXiv:hep-th/9206078.

[13] S. Elitzur, A. Giveon, D. Kutasov and E. Rabinovici, "From big bang to big crunch and beyond," JHEP 0206, 017 (2002) arXiv:hep-th/0204189.

[14] B. Craps, D. Kutasov and G. Rajesh, "String propagation in the presence of cosmological singularities," JHEP 0206, 053 (2002) arXiv:hep-th/0205101.

[15] Y. Hikida and T. Takayanagi, "On solvable time-dependent model and rolling closed string tachyon," Phys. Rev. D 70, 126013 (2004) arXiv:hep-th/0408124.

[16] N. Toumbas and J. Troost, "A time-dependent brane in a cosmological background," JHEP 0411, 032 (2004) arXiv:hep-th/0410007.

[17] C. W. Misner, "Taub-NUT space as a counterexample to almost anything," Relativity Theory and Astrophysics I: Relativity and Cosmology, ed. J. Ehlers, Lectures in Applied Mathematics, Volume 8, American Mathematical Society, 160 (1967).

[18] M. Berkooz, B. Craps, D. Kutasov and G. Rajesh, "Comments on cosmological singularities in string theory," JHEP 0303, 031 (2003) arXiv:hep-th/0212215. 
[19] M. Berkooz and B. Pioline, "Strings in an electric field, and the Milne universe," JCAP 0311, 007 (2003) arXiv:hep-th/0307280.

[20] M. Berkooz, B. Pioline and M. Rozali, "Closed strings in Misner space: Cosmological production of winding strings," JCAP 0408, 004 (2004) arXiv:hep-th/0405126.

[21] M. Berkooz, B. Durin, B. Pioline and D. Reichmann, "Closed strings in Misner space: Stringy fuzziness with a twist," JCAP 0410, 002 (2004) arXiv:hep-th/0407216.

[22] B. Durin and B. Pioline, "Closed strings in Misner space: A toy model for a big bounce?," arXiv:hep-th/0501145.

[23] S. W. Hawking and G. F. R. Ellis, "The large scale structure of space-time," Cambridge University Press (1973).

[24] M. S. Costa, C. A. R. Herdeiro, J. Penedones and N. Sousa, "Hagedorn transition and chronology protection in string theory," arXiv:hep-th/0504102.

[25] S. W. Hawking, "The chronology protection conjecture," Phys. Rev. D 46, 603 (1992).

[26] H. Yang and B. Zwiebach, "Rolling closed string tachyons and the big crunch," arXiv:hep-th/0506076.

[27] L. Cornalba and M. S. Costa, "Unitarity in the presence of closed timelike curves," arXiv:hep-th/0506104.

[28] J. McGreevy and E. Silverstein, "The tachyon at the end of the universe," arXiv:hep-th/0506130.

[29] G. T. Horowitz, "Tachyon condensation and black strings," arXiv:hep-th/0506166.

[30] B. Craps, S. Sethi and E. P. Verlinde, "A matrix big bang," arXiv:hep-th/0506180.

[31] M. Berkooz, Z. Komargodski, D. Reichmann and V. Shpitalnik, "Flow of geometries and instantons on the null orbifold," arXiv:hep-th/0507067.

[32] M. Pawlowski, W. Piechocki and M. Spalinski, "Winding strings in singular spacetimes," arXiv:hep-th/0507142.

[33] S. Kawai, E. Keski-Vakkuri, R. G. Leigh and S. Nowling, "Brane decay from the origin of time," arXiv:hep-th/0507163.

[34] Y. Hikida, R. R. Nayak and K. L. Panigrahi, "D-branes in a big bang/big crunch universe: Nappi-Witten gauged WZW model," JHEP 0505, 018 (2005) arXiv:hep-th/0503148.

[35] A. Hashimoto and S. Sethi, "Holography and string dynamics in time-dependent backgrounds," Phys. Rev. Lett. 89, 261601 (2002) arXiv:hep-th/0208126.

[36] M. Alishahiha and S. Parvizi, "Branes in time-dependent backgrounds and AdS/CFT correspondence," JHEP 0210, 047 (2002) arXiv:hep-th/0208187. 
[37] L. Dolan and C. R. Nappi, "Noncommutativity in a time-dependent background," Phys. Lett. B 551, 369 (2003) arXiv:hep-th/0210030.

[38] R. G. Cai, J. X. Lu and N. Ohta, "NCOS and D-branes in time-dependent backgrounds," Phys. Lett. B 551, 178 (2003) arXiv:hep-th/0210206.

[39] K. Okuyama, "D-branes on the null-brane," JHEP 0302, 043 (2003) arXiv:hep-th/0211218.

[40] D. Kutasov, "D-brane dynamics near NS5-branes," arXiv:hep-th/0405058.

[41] Y. Nakayama, Y. Sugawara and H. Takayanagi, "Boundary states for the rolling D-branes in NS5 background," JHEP 0407, 020 (2004) arXiv:hep-th/0406173.

[42] D. A. Sahakyan, "Comments on D-brane dynamics near NS5-branes," JHEP 0410, 008 (2004) arXiv:hep-th/0408070.

[43] B. Chen, M. Li and B. Sun, "D-brane near NS5-branes: With electromagnetic field," JHEP 0412, 057 (2004) arXiv:hep-th/0412022.

[44] Y. Nakayama, K. L. Panigrahi, S.-J. Rey and H. Takayanagi, "Rolling down the throat in NS5-brane background: The case of electrified D-brane," JHEP 0501, 052 (2005) arXiv:hep-th/0412038.

[45] Y. Nakayama, S.-J. Rey and Y. Sugawara, "D-brane propagation in two-dimensional black hole geometries," arXiv:hep-th/0507040

[46] J. D. E. Grant, "Cosmic strings and chronology protection," Phys. Rev. D 47, 2388 (1993) arXiv:hep-th/9209102.

[47] P. H. Ginsparg, "Applied conformal field theory," arXiv:hep-th/9108028.

[48] J. G. Russo and A. A. Tseytlin, "Constant magnetic field in closed string theory: An Exactly solvable model," Nucl. Phys. B 448, 293 (1995) arXiv:hep-th/9411099.

[49] M. Kato and K. Ogawa, "Covariant quantization of string based on BRS invariance," Nucl. Phys. B 212, 443 (1983).

[50] J. Polchinski, "String theory," Cambridge University Press (1998).

[51] M. Asano and M. Natsuume, "The no-ghost theorem for string theory in curved backgrounds with a flat timelike direction," Nucl. Phys. B 588, 453 (2000) arXiv:hep-th/0005002.

[52] M. Asano and M. Natsuume, "The no-ghost theorem in curved backgrounds with a timelike $u(1)$ : NSR string," Nucl. Phys. B 672, 39 (2003) arXiv:hep-th/0303051.

[53] R. Bott and L.W. Tu, "Differential forms in algebraic topology," Springer-Verlag (1982).

[54] P. Bouwknegt, J. G. McCarthy and K. Pilch, "BRST analysis of physical states for 2D gravity coupled to $c \leq 1$ matter," Commun. Math. Phys. 145, 541 (1992). 
[55] N. Seiberg and E. Witten, "String theory and noncommutative geometry," JHEP 9909, 032 (1999) arXiv:hep-th/9908142.

[56] C. Bachas, "D-brane dynamics," Phys. Lett. B 374, 37 (1996) arXiv:hep-th/9511043.

[57] M. Billo, P. Di Vecchia and D. Cangemi, "Boundary states for moving D-branes," Phys. Lett. B 400, 63 (1997) arXiv:hep-th/9701190.

[58] J. S. Schwinger, "On gauge invariance and vacuum polarization," Phys. Rev. 82, 664 (1951).

[59] C. Bachas and M. Porrati, "Pair creation of open strings in an electric field," Phys. Lett. B 296, 77 (1992) arXiv:hep-th/9209032.

[60] J. L. Karczmarek, H. Liu, J. Maldacena and A. Strominger, "UV finite brane decay," JHEP 0311, 042 (2003) arXiv:hep-th/0306132.

[61] M. Billo, B. Craps and F. Roose, "Orbifold boundary states from Cardy's condition," JHEP 0101, 038 (2001) arXiv:hep-th/0011060.

[62] C. G. Callan, C. Lovelace, C. R. Nappi and S. A. Yost, "Loop corrections to superstring equations of motion," Nucl. Phys. B 308, 221 (1988).

[63] N. Lambert, H. Liu and J. Maldacena, "Closed strings from decaying D-branes," arXiv:hep-th/0303139.

[64] D. Gaiotto, N. Itzhaki and L. Rastelli, "Closed strings as imaginary D-branes," Nucl. Phys. B 688, 70 (2004) arXiv:hep-th/0304192.

[65] A. Sen, "Rolling tachyon," JHEP 0204, 048 (2002) arXiv:hep-th/0203211.

[66] A. Sen, "Tachyon matter," JHEP 0207, 065 (2002) arXiv:hep-th/0203265.

[67] A. Adams, J. Polchinski and E. Silverstein, "Don't panic! Closed string tachyons in ALE space-times," JHEP 0110, 029 (2001) arXiv:hep-th/0108075.

[68] P. Di Vecchia and A. Liccardo, "D branes in string theory. I," NATO Adv. Study Inst. Ser. C. Math. Phys. Sci. 556, 1 (2000) arXiv:hep-th/9912161.

[69] P. Di Vecchia and A. Liccardo, "D-branes in string theory. II," arXiv:hep-th/9912275.

[70] N. Birrel and P. Davies, "Quantum fields in curved space," Cambridge University Press (1984).

[71] S. Minwalla, M. Van Raamsdonk and N. Seiberg, "Noncommutative perturbative dynamics," JHEP 0002, 020 (2000) arXiv:hep-th/9912072.

[72] J. Polchinski, "Evaluation of the one loop string path integral," Commun. Math. Phys. 104, 37 (1986). 